Research Paper

\title{
NDRG1 regulates Filopodia-induced Colorectal Cancer invasiveness via modulating CDC42 activity
}

Batuer Aikemu ${ }^{1,2,3^{*}}$, Yanfei Shao ${ }^{1,2,3^{*}}$, Guang Yang ${ }^{1,2,3^{*}}$, Junjun Ma1,2, Sen Zhang ${ }^{1,2}$, Xiao Yang ${ }^{1,2}$, Hiju Hong ${ }^{1,2}$, Galiya Yesseyeva ${ }^{1,2,3}$, Ling Huang ${ }^{1,2,3}$, Hongtao Jia ${ }^{1,2,3}$, Chenxing Wang ${ }^{1,2,3}$, Lu Zang ${ }^{1,2}$, Jing Sun ${ }^{1,2}$ and Minhua Zheng $1,2 \llbracket$

1. Department of General Surgery, Ruijin Hospital, Shanghai Jiao Tong University School of Medicine, Shanghai, China.

2. Shanghai Minimally Invasive Surgery Center, Ruijin Hospital, Shanghai Jiao Tong University School of Medicine, Shanghai, China.

3. Department of Surgery, Shanghai Key Laboratory of Gastric Neoplasms, Shanghai Institute of Digestive Surgery, Ruijin Hospital, Shanghai Jiao Tong University School of Medicine, Shanghai, China.

*First authors with equal contributions to this work.

$\bowtie$ Corresponding authors: Prof. Minhua Zheng (E-mail: zmhtiger@yeah.net), Dr. Jing Sun (E-mail: jingsun1982@sina.cn) \& Dr. Lu Zang (E-mail: zanglu@yeah.net) contributed equally to this work.

(C) The author(s). This is an open access article distributed under the terms of the Creative Commons Attribution License (https://creativecommons.org/licenses/by/4.0/). See http://ivyspring.com/terms for full terms and conditions.

Received: 2020.12.02; Accepted: 2021.04.01; Published: 2021.04.17

\begin{abstract}
$\mathrm{N}$-myc downstream regulated gene-1 (NDRG1) has been identified as a putative metastasis suppressor gene and proved to be a key player in cancer spreading and proliferation in our previous work. However, the effects of NDRGI on tumor invasion and the mechanisms behind it are rarely understood. Here we provided in silico evidence that NDRGI plays a crucial role in actin reorganization in colorectal cancer (CRC). Through in vitro experiments, we next observed filopodia formation was altered in NDRG1-modified cell lines, while cell division cycle-42 (CDC42) displayed excessive activation in NDRG 1 -silenced cells. Mechanistically, NDRG 1 loss disrupts the binding between RhoGDIa and CDC42 and triggers the activation of CDC42 and the downstream cascades PAK1/Cofilin, thereby promotes the formation of filopodia and invasiveness of CRC. The knockdown of NDRGl led to enhanced dissemination of CRC cells in vivo and correlates with active CDC42 expression. Using clinical sample analysis, we found an elevated level of active CDC42 in patients with advanced T stage, and it was negatively related to NDRG 1 expression. In sum, these results uncover a mechanism utilized by NDRG 1 to regulate CDC42 activity in coordinating cytoskeleton reorganization, which was crucial in cancer invasion.
\end{abstract}

Key words: colorectal cancer, NDRG1, cytoskeleton, invasion, CDC42

\section{Introduction}

Colorectal cancer (CRC) is the third most commonly diagnosed cancer in both men and women worldwide [1], and its incidence and mortality rates in China over the past decades have been on an upward trend [2]. In the early phase of metastatic spread, CRC cells rely much on the actin cytoskeleton's dynamic reorganization to change shape to gain mechanical support that promotes cell motility and invasion [3].

$\mathrm{N}$-myc downstream regulated gene-1 (NDRG1), identified as a metastasis suppressor recently, has been proven to be a key player in affecting cancer proliferation, spreading, cell adhesion, and autophagy [4-6]. Our team has recently reported new mechanisms of NDRG1 in regulating the proliferation of CRC cells [7] and inhibiting EMT through its interaction and promotion of caveolin-1 [8] and found out its potential role in regulating stress fibers assembly [9]. Despite the limited studies that displayed possible functions of NDRG1 during actinfilament assembly in prostate and oral squamous cell cancers $[10,11]$, no adequate explanation has been given for the underlying mechanism.

Actin-cytoskeleton reorganization regulates cell morphological changes, namely lamellipodia and filopodia formation, and results in the directional migration and invasion of cancer cells [12]. As one of the critical factors in tumor metastasis, the activation of cytoskeletal proteins triggers the beginning of an 
invasive or metastatic process [13] and sometimes facilitates the survival of extravasated tumor cells [14]. This migratory strategy of cancer cells to acquire optimum shape and obtain protrusive force is thought to be controlled by Rho GTPases, a family of small signaling $G$ proteins $[15,16]$. Of the numerous functions Rho GTPases have shown in both normal and cancerous cells, the actin cytoskeleton's remodeling is one of the most remarkable. It could regulate the dynamics of cell morphology and motility by interacting with various downstream signaling molecules. For example, filopodium is an actin-rich protruding structure that extends from the plasma membrane in a fingerlike manner, and it has been thought to be largely influenced by the Rho GTPases activation [17]. It serves as a hub where diverse cellular processes orchestrate and is closely involved in cell invasion and migration both in vitro and in vivo [17].

Notably, despite the preliminary evidence indicating an association between NDRG1 and actin reorganization, the precise mechanism of the metastasis suppressor in intervening cell protrusion remains unclear. We therefore investigated whether NDRG1 played a role in actin structure remodeling of CRC and, if so, what the functional role and clinical importance are. To the best of our knowledge, this work is the first attempt to demonstrate that the suppression of the CDC42/PAK1/Cofilin pathway, a key regulator of filopodia formation, underlies the ability of NDRG1 to inhibit CRC invasion. Mechanistically, NDRG1 could stabilize the RhoGDIa-CDC42 interaction and thereby affect the oscillation of CDC42 activation. Thus, the potential influence of NDRG1 in cytoskeleton reorganization via regulating actin filament depolymerization might give a better insight into the crucial role that NDRG1 plays in cancer invasiveness.

\section{Materials and Methods}

\section{Cell culture and transfection}

The HCT116 and RKO colon cancer cell lines were purchased from the American Type Culture Collection (ATCC, Manassas, VA). All cells were cultured in RPMI 1640-medium supplemented with $10 \%$ fetal bovine serum (FBS) and maintained at a 37 ${ }^{\circ} \mathrm{C}$ incubator with a $5 \% \mathrm{CO}_{2}$ humidified atmosphere. The NDRG1 overexpression and knockdown clones were established as described previously [9]. For transient transfection, cells were seeded in a 6-well culture plate $24 \mathrm{~h}$ before transfection; then cells were transfected with corresponding siRNA or vector, using Lipofectamine 2000 (Invitrogen), according to the manufacturer's instructions. siRNA duplexes used were as follows: siCDC42\#1: sense: 5'-CCUGAAGGC UGUCAAGUAUTT-3', antisense: 5'-AUACUUGACA GCCUUCAGGTT-3'; siCDC42\#2: sense: 5'-GCUUGU UGGGACUCAAAUUTT-3', antisense: 5'-AAUUUGA GUCCCAACAAGCTT-3'; siCDC42\#3: sense: 5'-CCG CUGAGUUAUCCACAAATT-3', antisense: 5'-UUUG UGGAUAACUCAGCGGTT-3'.

\section{RNA isolation and quantitative real-time qRT-PCR analysis}

RNA extraction and qRT-PCR analysis were performed as described previously [8]. The primer sequences of all genes were listed as follows:

NDRG1 (5'-CTGCACCTGTTCATCAATGC-3' and 5'-AGAGAAGTGACGCTGGAACC-3'); GAPDH (5'-TTCAACAGCAACTCCCACTCTT-3' and 5'-TGGT CCAGGGTTTCTTACTCC-3'); FGD1 (5'- AGATATAC AGCACCAAGGGTTC-3' and 5'-CACTACATGGAG AAGGGTGG-3'); PLEKHG2 (5'-GAACTGTTTTCTGG GAGCAATC-3' and 5'-TGGAAGTCTGTGAATGATA CCC-3'); PREX1 (5'-GCAATATGTCACCCAGATC AAC-3' and 5'-GTAGGAGTCGCGATAACTC ATG-3'); TUBA (5'-CAAAGTCAAGACCTCGTCA AAG-3' and 5'-GGATCTTGAGTTTCTGATTGGC-3'); PLEKHG4 (5'-TGTCCAGGAAATTCCAGTTACC-3' and 5'-GACTGAGGAGCTTTCTACTGTG-3'); ITSN1 (5'-TGGAGAAGTTCAAGGTCAGA-3' and 5'-TGTCA GCAGCTCAGACTCCA-3'); ITSN2 (5'-GCGCAGTCT CTGATTGATTTAG-3' and 5'- GAAGAAGGGCATTT CTAGCTTG-3'); ECT2 (5'-CTAAAGATGCTGTGTCG ACATG-3' and 5'-TTGCTCTTGATGCTCTACT CAA-3'); PLEKHG1 (5'-CTCTGAAACACTCGCTGCC TCTG-3' and 5'-AGCATCAAGCACCACATCATA GCC-3'); FGD4 (5'-TGGGCTATGTGGTGGATGAAA TGC-3' and 5'-GCCACTTCTGCTTCAGTTCCT CAC-3'); ARHGAP12 (5'-GAACAGGTCTTATTCGTG ATGC-3' and 5'-CAAATGAGCGTGTCCTATT CTG-3'); ARHGAP26 (5'- ACCAACAAATTCATCAA GGAGC-3' and 5'-TCAATCATCCGTATCCGTT CAT-3').

\section{Scanning electron microscopy (SEM)}

Cells seeded on coverslips for $36 \mathrm{~h}$ were fixed in $2.5 \%$ glutaraldehyde for $2 \mathrm{~h}$ at room temperature, washed in PBS for three times, dehydrated with graded ethanol at $4{ }^{\circ} \mathrm{C}$, soaked in $100 \%$ acetone for 20 min, and $100 \%$ isoamyl acetate for $15 \mathrm{~min}$ and propylene epoxide for $20 \mathrm{~min}$ at $45^{\circ} \mathrm{C}$. The coverslips were then vacuumed and spray-coated with metal foil and put under the Field Emission Scanning Electron Microscopy (SU8010, Hitachi, Japan) for observation.

\section{Immunofluorescence staining and evaluation}

Cells seeded on coverslips for more than $24 \mathrm{~h}$ were fixed in $4 \%$ formaldehyde for $30 \mathrm{~min}$ at room temperature, then permeabilizated with $0.1 \%$ Triton 
X-100 for $30 \mathrm{~min}$. After that, cells were treated with the primary antibody overnight at $4^{\circ} \mathrm{C}$, followed by incubation with species-specific secondary antibody for two hours at room temperature. The coverslips were mounted using an antifade mounting solution containing 4,6-diamidino- 2-phenylindole (DAPI; P36935, Invitrogen) after three-time washing with phosphate-buffered saline (PBS). Actin was stained with Myo10 (Rabbit, HPA024223, Sigma-Aldrich) and Rhodamine Phalloidin (R415, Invitrogen) following the manufacturer's instructions. Confocal images were captured using ZEISS LSM 880 confocal microscope with Airyscan (Carl Zeiss, Germany) or Leica TCS SP8 confocal microscope (Leica, Switzerland), and fluorescence quantification was measured as described previously [9]. Co-localization analysis was performed via Leica Application Suite X (LAS X).

\section{Immunoblotting, GST-pull down, and immunoprecipitation assay}

Briefly, in the immunoblotting assay, lysates of cell samples were separated by SDS-PAGE gel, transferred to a PVDF membrane, blocked with 5\% milk, and incubated with primary antibodies, then species-specific secondary antibodies. The primary antibodies included:

NDRG1 (Rabbit, Cat.ab124689, Abcam); GAPDH (Mouse, Cat. 60004-1-Ig, Proteintech); Myo10 (Rabbit, HPA024223, Sigma-Aldrich); RAC1 (Rabbit, Cat.ab155938, Abcam); CDC42 (Rabbit, Cat.ab187643, Abcam), CDC42 (Mouse, Cat.sc-8401, Santa Cruz); CDC42GTP (Mouse, Cat.26905, Neweast Bio); Phospho-Cofilin (Thr423) (Rabbit, Cat.2601, Cell Signaling Technology); PAK1 (Rabbit, Cat.2602, Cell Signaling Technology); Phospho-Cofilin (Ser3) (Rabbit, Cat.3313T, Cell Signaling Technology); Cofilin (Rabbit, Cat.5175T, Cell Signaling Technology); N-WASP (Rabbit, Cat.4848, Cell Signaling Technology); Profilin-1 (Rabbit, Cat.3246, Cell Signaling Technology); ARP2 (Rabbit, ab47654, Abcam); ARP3 (Rabbit, Cat.4738, Cell Signaling Technology); PLEKHG2 (Rabbit, Cat.ab180156, Abcam); RhoGDIa (Rabbit, Cat.ab133248, Abcam), RhoGDIa (Rabbit, Cat.2564, Cell Signaling Technology).

GST-pull down assay to detect active CDC42 and RAC1 was carried out as the protocol of Active CDC42 Detection Kit (Cat.8819, Cell Signaling Technology) and Active RAC1 Detection Kit (Cat.8815, Cell Signaling Technology).

Immunoprecipitation was performed as described previously [7]. Briefly, cell lysis containing $300 \mathrm{mg}$ protein was incubated with the CDC42 antibody (Rabbit, Cat.ab187643, Abcam) overnight at
$4{ }^{\circ} \mathrm{C} .30 \mu \mathrm{l}$ of $\mathrm{A} / \mathrm{G}$ magnetic beads (Pierce Crosslink Magnetic IP/Co-IP Kit, Cat.88805) from Thermo Fisher Scientific (MA, USA) was then added into the mixture and incubated $4 \mathrm{~h}$ at $4{ }^{\circ} \mathrm{C}$. The beads were washed, and a low $\mathrm{pH}$ elution buffer is used to dissociate bound antigen from the antibodycrosslinked beads. A neutralization buffer is applied to prevent precipitation of the isolated antigen. Resuspended in loading buffer and incubated over 90 ${ }^{\circ} \mathrm{C}$ for $10 \mathrm{~min}$, the supernatant was then separated on a $12.5 \%$ Bis-Tris gel. Secondary antibody for IP detection (Veriblot, Cat.ab131366, Abcam) was used to detect the specific protein in the immunoblotting assay.

\section{Invasion assay}

Invasion assay using Transwell chambers $(8 \mu \mathrm{m}$ for 24-well plate; Corning Costar, NY, USA) was performed as previously described [18], and the incubating time of different cell-type was as indicated in the results.

\section{Peritoneal metastatic xenograft model}

Male BALB/c nude mice at the age of about four weeks (Charles River, Beijing, China) were housed at a specific pathogen-free environment and randomly divided into two groups (5 mice in each) (Research Center of Experimental Medicine, Shanghai Jiao Tong University School of Medicine Affiliated Ruijin Hospital). HCT116 sh-NDRG1 cells and the negative control cells were lentivirally transduced firefly luciferase fusion vector (GeneChem, Shanghai, China), and stably transfected cells were selected with $10 \mu \mathrm{g} / \mathrm{ml}$ puromycin. The colon cancer cells were trypsinized and resuspended in $100 \mu \mathrm{l}$ PBS which contained $5 \times 10^{5}$ cells and injected into the mice's abdomen. Before the euthanasia, tumor distribution and mass were assessed by bioluminescence imaging (Caliper Life Sciences, USA) in the fourth week. The sample size was calculated with Spectrum living image software, and subsequently, peritoneal foci were checked by gross specimens and microscopy. All experiments adhered to the NIH Guide for the Care and Use of Laboratory Animals.

\section{Clinical patient tissue microarray and IHC analysis}

The microarrays, which contained 86 colorectal cancer and paired adjacent normal paraffinembedded specimens, were supplied by the Shanghai Minimally Invasive Surgery Center of Ruijin Hospital (Shanghai, China) following the guidelines by the Ethics Committee of Ruijin Hospital. Written and informed consent of all the cases was signed before the study. After immunohistochemical assay was conducted as described [18], two independent 
pathologists analyzed the expression levels of target proteins. The overall score was determined by multiplying the cellular staining proportion $(0=0 \%, 1$ $=\leq 25 \%, 2=26$ to $50 \%, 3=51$ to $75 \%, 4=76$ to $100 \%$ positive) and intensity ( $0=$ no staining, $1=$ weak, $2=$ moderate, 3 = strong). All cases were delimited into two equivalent subgroups by the median score of CDC42GTP. NDRG1 expression was considered high if the overall score was greater than six and low if it was six or less.

\section{Statistical analysis}

Experimental data are presented as the mean \pm S.D.; two-tailed Student's t-tests with unequal variance were performed using GraphPad Prism 8.0 for macOS (GraphPad Software). The chi-square test or Fisher's exact test was used for categorical variable comparison by IBM SPSS 22.0 for macOS (SPSS INC.). The TCGA dataset results were carried out by using the package in $\mathrm{R}$ version (http://www.r-project.org). Differences were considered statistically significant when $P$ values were less than 0.05 .

\section{Results}

\section{NDRG 1 loss results in increased filopodia formation and invasiveness of CRC cell lines}

We previously demonstrated a novel thiosemicarbazone iron chelator regulating actinfilament reorganization via a mechanism involving NDRG1 [9]. To further clarify the functional role of NDRG1 in CRC, we first compared the NDRG1-lowexpression (lower half) colorectal cancer samples with the NDRG1-high-expression (higher half) samples in silico using The Cancer Genome Atlas (TCGA). We extracted 1786 differential expressed genes (DEGs) with criteria of $\mathrm{P}<0.05$ and $|\log \mathrm{FC}|>1$ (Figure S1A). Gene Ontology (GO) and Kyoto Encyclopedia of Genes and Genomes (KEGG) enrichment analysis revealed multiple DEGs involved in focal adhesion, extracellular matrix (ECM) receptor interaction, regulation of actin cytoskeleton, and binding of integrin and actin (Figure S1B-C).

To obtain a broader understanding of NDRG1's role in actin remodeling and cell aggressiveness, we first established HCT116 and RKO CRC cell lines constitutively and stably express or knockdown NDRG1 (Figure S2A-B, P<0.001). With different traits in their cell phenotype and aggressiveness [19], these two cell models were introduced to show the consistency of different cell lines' responses. Next, we applied the immunofluorescence staining to visualize the cytoskeleton morphology. Co-stained with filopodia-specific binder Myosin-10 (Myo10), the structure of F-actin showed a remarkable alteration in the filopodial protrusion in the NDRG1-modified CRC cells. We observed clear, thin, and elongated protrusions outside the plasma membrane of both HCT116 and RKO cells, which matched the definition of filopodia [20] well. However, the plasma membrane protrusions were significantly decreased in number and weaker and shorter in size in NDRG1overexpressed cells than vector cells (Figure 1A-B). Moreover, compared with the relative sparser and shorter filopodia presented in control cells, the F-actin filaments were aggregated into thick, abundant, and elongated membrane protrusions in the plasma and generated polarity in sh-NDRG1 cells of both cell lines (Figure 1A-B). We next applied scanning electron microscopy and observed that NDRG1-knockdown cells exhibited more elongated and enlarged filopodia, whereas relatively shorter and fewer microspikes presented in NDRG1-overexpression cells compared with respective control cells (Figure S3). These results collectively indicate that less NDRG1 might facilitate cytoskeleton rearrangement in colorectal cancer cells by promoting filopodia formation.

It is believed that during the event of invasive cancer cells penetrating the underlying stroma, filopodia play the role of pathfinder, which could degrade the basement membrane and then guide the cell body entering the compartment [21]. We thereby observed the invasiveness of the CRC cells through matrigel-coated transwells and found that NDRG1-overexpressing showed an inhibitory effect on cell invasion while silencing NDRG1 distinctly upregulated cell invasion (Figure $1 \mathrm{C}, \mathrm{P}<0.001$ or $\mathrm{P}<0.01)$. This phenomenon was consistent in both cell types and could reflect the previous findings of morphological alteration on cancer cells.

\section{NDRG 1 negatively regulates filopodia formation by inhibiting CDC42 activation}

The small Rho GTPases are essential regulators of actin cycling and cytoskeleton reorganization. Activation of CDC42 has been proven to trigger filopodia formation and forward movement of motile cells, whereas RAC1 activity drives the extension of lamellipodia, which are broad and flat membrane protrusions always show up at the leading edge [12, $13,22]$. They cycle between two conformational states, namely active state (bounding to GTP) and inactive state (bounding to GDP), and regulate many aspects of cancerous behavior [23]. To further investigate whether the filopodia diversity observed in NDRG1-modified CRC cells was realized through the regulation of Rho GTPase family members, we detected the expression of the two major cell protrusion regulators; however, no significant 
alteration of the two proteins' expression was observed in either cell line (Figure 2A). Since the vital signaling function of RAC1 and CDC42 in cytoskeletal reorganization intensively depends on their biological activities, we next evaluated the transformation of active RAC1 and CDC42. Detection of RAC1 demonstrated no significant change, whereas CDC42 activation (i.e., CDC42GTP) in both cell lines was dramatically $(\mathrm{P}<0.05)$ inhibited by over-expressed NDRG1 and markedly $(\mathrm{P}<0.01$ or $\mathrm{P}<0.05)$ enhanced when NDRG1 was silenced (Figure 2A). These indicated that NDRG1 might reduce the activity of CDC42, to regulate actin cytoskeletal dynamics in colorectal cancer cells. In support of this hypothesis, we further performed immunofluorescence experiments to confirm the alteration of CDC42GTP in both cell lines. The fluorescence intensity of CDC42GTP was decreased $(\mathrm{P}<0.01$ or $\mathrm{P}<0.05)$ and increased $(\mathrm{P}<0.001)$ in response to NDRG1 overexpression and knockdown, respectively (Figure 2B). These accumulative data suggest that NDRG1 suppresses the filopodia formation by diminishing the activity of CDC42.

\section{NDRG 1-mediated filopodia rearrangement is regulated through a PAK1/Cofilin dependent pathway}

To elucidate the cell invading mechanism triggered by over-activation of CDC42GTP, we next examined the downstream axis that targets actin filament reorganizing. Among the signaling pathways implicated in the CDC42-mediated filopodia formation, Wiskott-Aldrich syndrome protein (WASP) and insulin receptor tyrosine kinase substrate of $53 \mathrm{kDa}$ (IRSp53) are canonical downstream targets, which could be activated by CDC42 and promote actin nucleation and polymerization by interacting with actin-related protein 2/3 (ARP2/3) complex[24]. Therefore, we asked through which pathway CDC42 impacted the cytoskeleton remodeling in an NDRG1-mediated manner. However, examining the protein level of the candidates above showed no significant change (Figure S4). Given that the ARP2/3-targeted signaling mainly contributes to the actin polymerization, we supposed that the cytoskeleton reorganizing might have been mediated via the process of actin dissembling.

Phosphorylation of p21-activated kinase 1 (PAK1), another downstream target for CDC42, could inhibit Cofilin's function and thereby triggers the debranching of actin filaments [25]. To explore whether depolymerization was the reason for filopodial response upon NDRG1/CDC42 signaling, we next examined the activity of related markers involved. As shown in Figure 3A, total PAK1 or
Cofilin expression was not affected when NDRG1 was overexpressed or knocked down in either HCT116 or RKO cells. Nonetheless, overexpression of NDRG1 significantly $(\mathrm{P}<0.01$ or $\mathrm{P}<0.05)$ decreased the phosphorylation levels of PAK1 (i.e., pPAK1/PAK1 ratio) and Cofilin (i.e., pCofilin/Cofilin ratio), while knockdown of endogenous NDRG1 led to a significant $(\mathrm{P}<0.01$ or $\mathrm{P}<0.05)$ enhancement of the phosphorylated form of PAK1 and Cofilin in both cell types (Figure $3 \mathrm{~A}$ ). The binding of RAC1/CDC42 to the PBD of p21-activated kinase (PAK) causes autophosphorylation and conformational changes in PAK, and it is known that phosphorylation of PAK1 at threonine 423 results in an increased PAK1 activity [26]. PAK1 plays an essential role in cytoskeletal rearrangements by activating LIM kinase, which subsequently phosphorylates Cofilin into an inactive form and thus decreases the depolymerization rates [27].

To uncover the role of CDC42 in the NDRG1mediated actin depolymerization signaling pathway, we next inhibited the cellular CDC42 process with the combined siRNA (Figure 3B). As expected, suppressing CDC42 in either NDRG1 knockdown or control cells could dramatically $(\mathrm{P}<0.001, \mathrm{P}<0.01$, or $\mathrm{P}<0.05)$ down-regulate the phosphorylation levels of PAK1 (Figure 3C) and reverse the NDRG1-silencinginduced pCofilin accumulation. Cofilin, a modulator that promotes the F-actin's debranching and severing, orchestrates cytoskeleton reorganization only in the non-phosphorylated form [28]. These results confirm that CDC42 was the key mediator in the NDRG1inhibited PAK/Cofilin signaling and cytoskeleton reorganization. Given that the role of PAK1/Cofilin in reforming filopodia was well characterized[29], we further assessed the reversal effect of depleting CDC42 in cell protrusion formation by measuring filopodial density and length by rhodaminephalloidin and Myo10 co-staining (Figure 3D). The quantification analysis corroborated our previous finding that loss of NDRG1 induced more aggressive growth of filopodia than in the control group. Moreover, the inhibition of CDC42 in sh-NDRG1/siCDC42 groups of both cell lines reversed the overgrowth dramatically (Figure 3D, P<0.001) compared with sh-NDRG1/si-Con groups. Moreover, the over-invasiveness of CRC cells induced by silencing NDRG1 was impeded when inhibiting CDC42 (Figure S5, $\mathrm{P}<0.001$ ). In sum, these results explain the phenotypic change in cancer cells and indicate an inhibitory role of NDRG1 in actin reorganization and cell invasiveness via a CDC42/PAK1/Cofilin dependent pathway. 
A

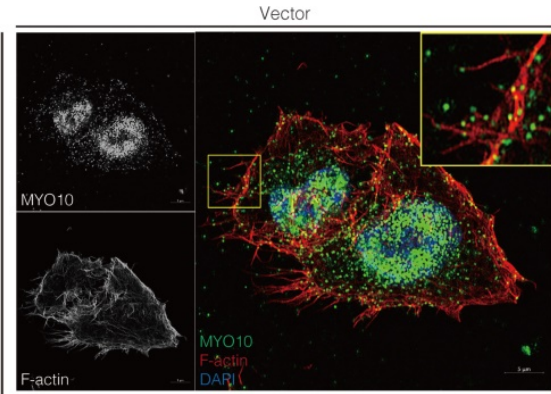

HCT116

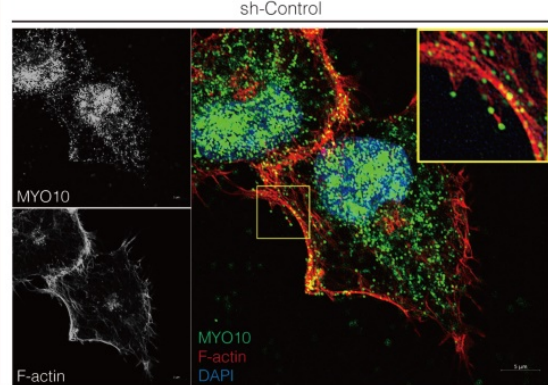

B

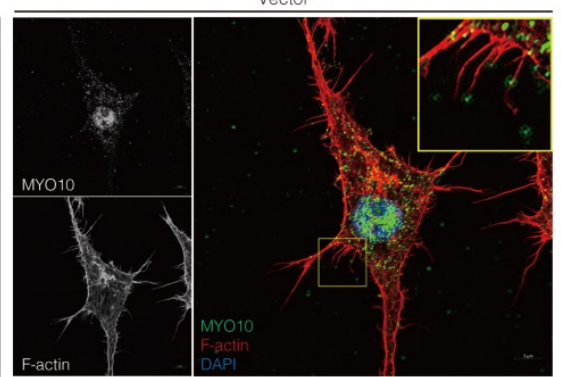

RKO

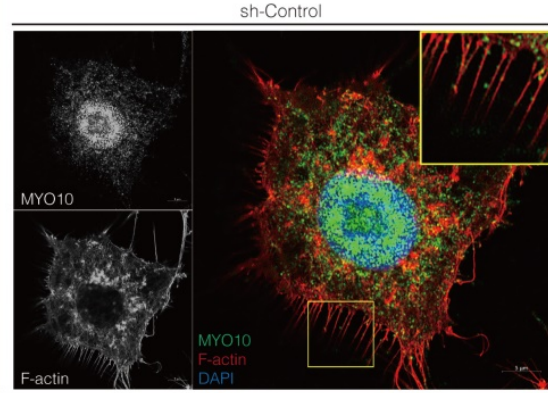

C
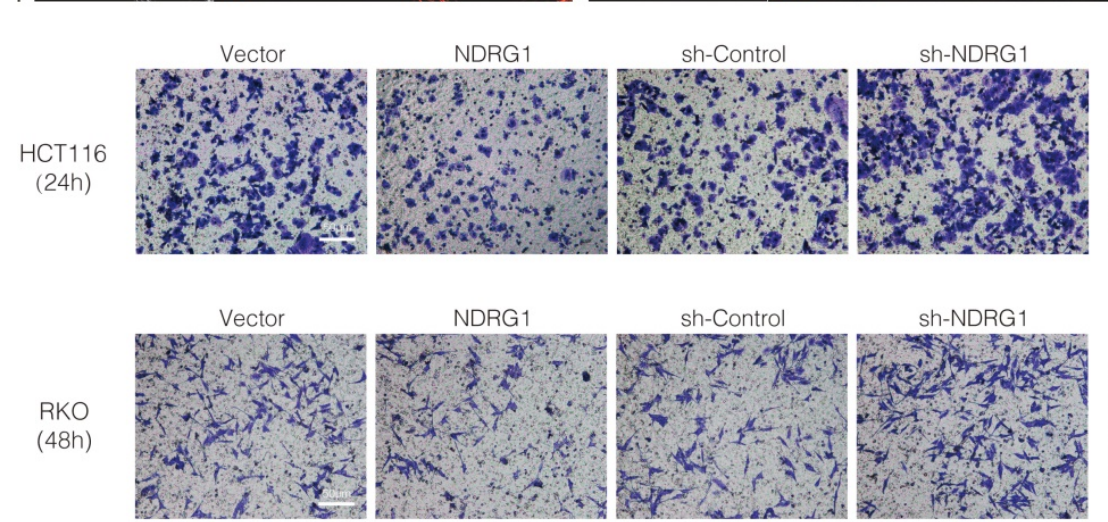

NDRG1
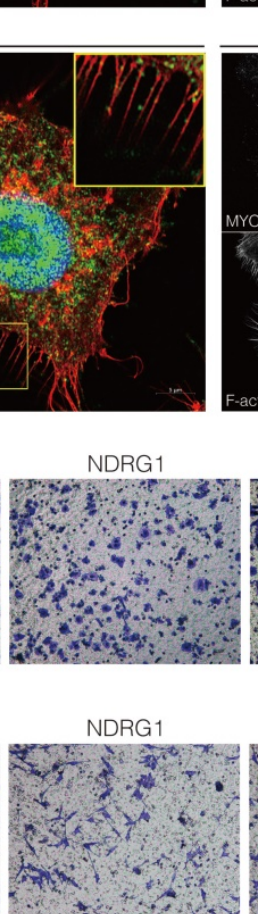

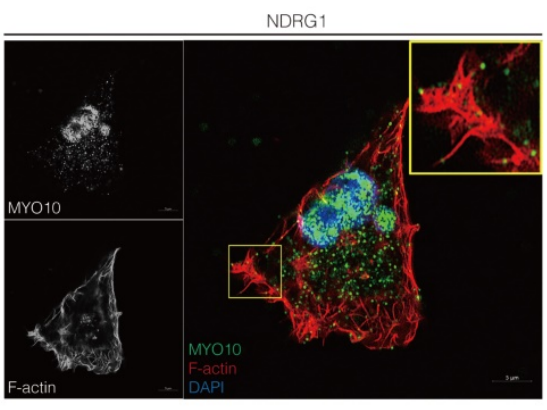

sh-NDRG1

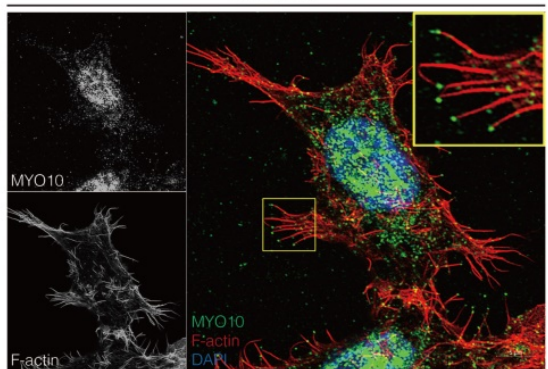

NDRG 1

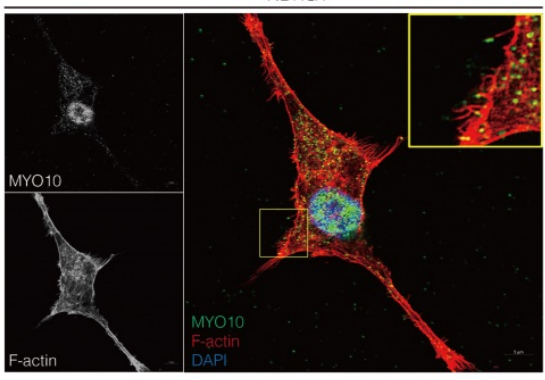

sh-NDRG1
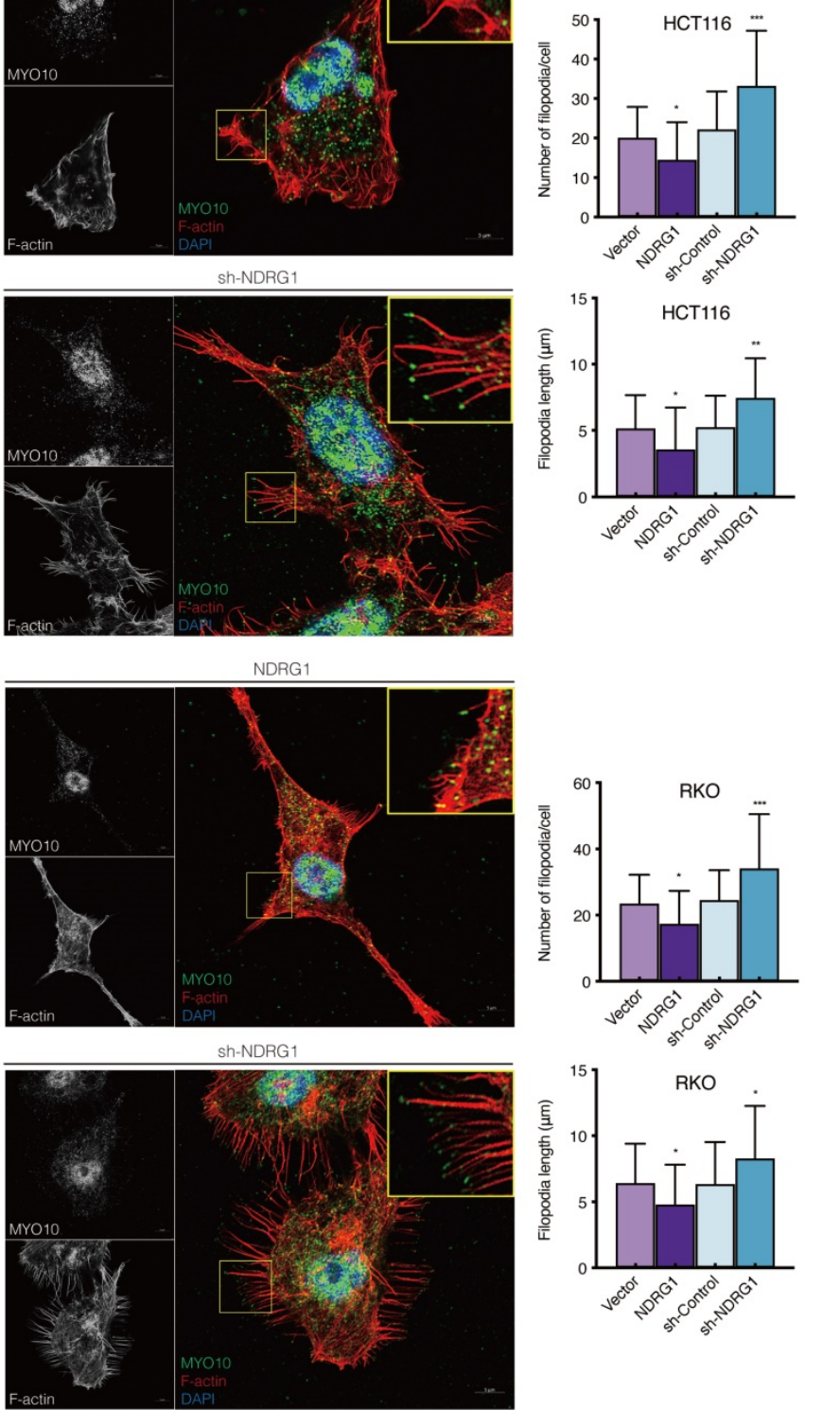
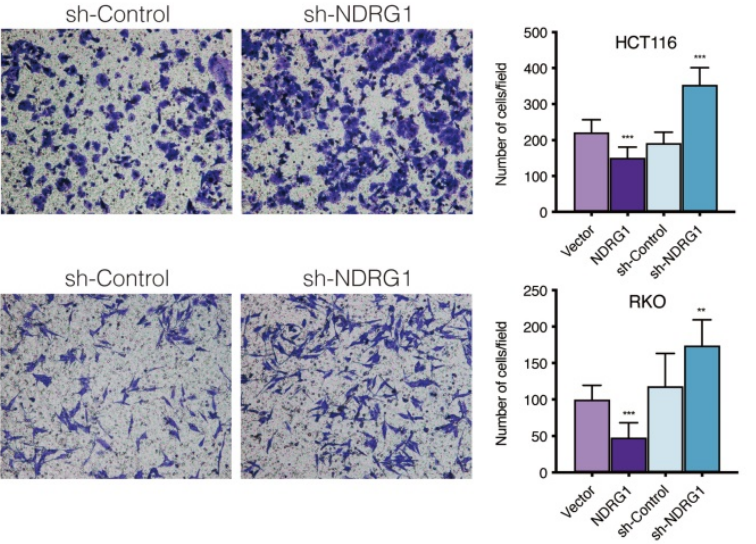

Figure 1. NDRG1 loss results in increased filopodia formation and invasiveness in CRC cells. A-B) Confocal images of the immunofluorescence staining of MYO10 (green) and rhodamine-phalloidin (red) accompanied by the cell nucleus (blue) in HCT116 (A) and RKO (B) cells. The insets show magnifications of MYO10-associated filopodial protrusions in the boxed areas. Quantification of the filopodial protrusions density and filopodia length is represented as mean \pm S.D.; $n>50$ cells from three biological repeats;

*P value $<0.05$, **P value $<0.01$, ***P value $<0.001$, relative to the respective control cells. Scale bar: $5 \mu \mathrm{m}$. C) Transwell invasion assay of $\mathrm{HCT} 116$ and RKO cells with NDRG 1 overexpressing or silencing after incubating for $24 \mathrm{~h}$ (HCT1 16 cells) or $48 \mathrm{~h}$ (RKO cells). Data represent the mean \pm S.D. of $3-5$ different experiments. **P value $<0.01$, ***P value $<0.001$, relative to the respective control cells. Scale bar: $50 \mu \mathrm{m}$. 
A
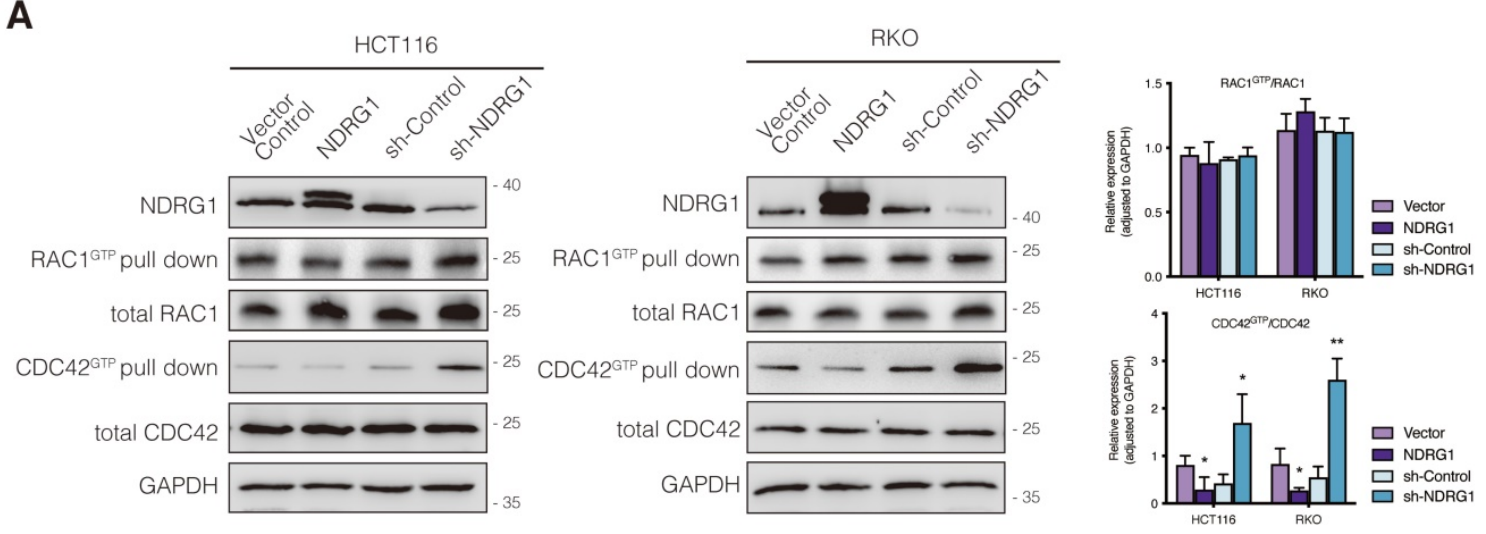

B
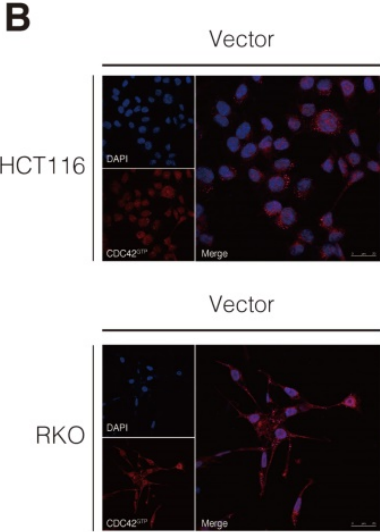

NDRG1

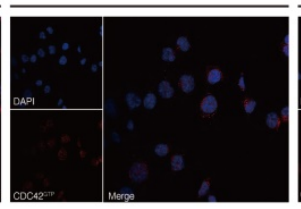

NDRG1

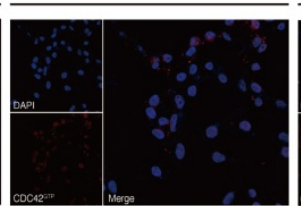

sh-Control

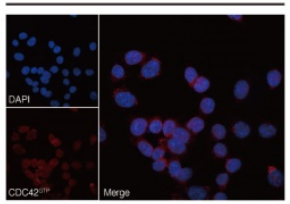

sh-Control

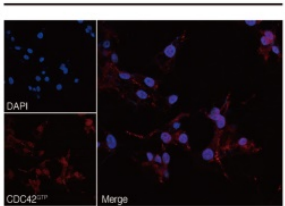

sh-NDRG1

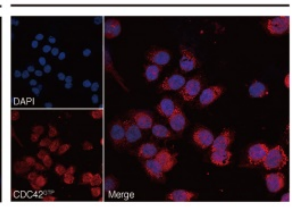

sh-NDRG1

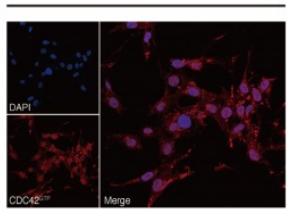

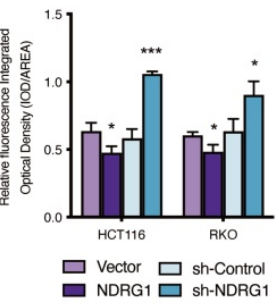

$\square$ Vector $\square$ sh-Control

Figure 2. Regulation of CDC42 activity by NDRG1 in CRC cells. A) Immunoblotting for total protein level or activated form of indicated Rho GTPase in NDRG 1-modified HCT1 16 and RKO cells. Results are representative of at least three biological repeats, and the values in histograms are represented by mean \pm S.D.; *P value $<0.05$, **P value $<0.01$, relative to the respective control cells. B) Confocal images were taken to show immunofluorescence staining of active-CDC42 (red) accompanied by the cell nucleus (blue) stained by DAPI in NDRGl overexpression and NDRG1 knockdown HCT116 and RKO cells relative to the control cells, respectively. Fluorescence quantification was performed by comparing the integrated optical density (IOD)/area value of active-CDC42 to the IOD/area value of the nucleus (DAPI) in the same image. Results are representative of three to five images from different visual fields, and the histogram values are mean $\pm S$.D. $* P$ value $<0.05$, $* * * P<0.001$, relative to the respective control cells. Scale bars: $25 \mu \mathrm{m}$.

\section{NDRG 1 inhibits CDC42 activation by stabilizing the RhoGDla-CDC42 binding}

Our study has revealed the CDC42 over-activation after NDRG1-depletion and consequently upregulated filopodia formation (Figure 3); however, the mediators between NDRG1 and the Rho GTPase were still obscure. To find out the previously known or predicted interactors of CDC42 that possibly modified the NDRG1-regulated cytoskeleton reorganization, we next referred to the STRING database (http://string-db.org/) to depict the protein-protein interaction network of CDC42 in human organisms. Among the top ten interactors, there are members of guanine nucleotide exchange factors (GEFs), GTPase-activating proteins (GAPs), and Rho GTPase dissociation inhibitors (RhoGDIs) families, which are well-recognized Rho GTPases modulators (Figure 4A). We first detected the mRNA expression levels of the canonical modulators in the NDRG1-overexpressed and -silenced CRC cells (Figure S6A). Despite the partial increase of PLEKHG2 shown in NDRG1-knockdown HCT116 cells, which was not consistent with the further immunoblotting examining (Figure S6B), we observed no remarkable changes in other potent GEFs or GAPs of CDC42. We then hypothesized the effect of the activation might have been occurring through the binding of RhoGDIs with CDC42, which leads to the stabilization of the inactive form of CDC42 [30].

To evaluate the binding of RhoGDIa and CDC42, co-immunoprecipitation (co-IP) was conducted. As shown, the binding was enhanced as NDRG1 was overexpressed; conversely, it decreased as NDRG1 was knockdown (Figure 4B) while the CDC42 quantity was consistent. Furthermore, the levels of RhoGDIa showed no significant difference in NDRG1-modified cells compared with the control cells (Figure 4C). We also detected the binding of NDRG1 to RhoGDIa and CDC42, but neither direct interaction was found through co-IP assay (data not shown). Further immunofluorescence staining confirmed that RhoGDIa has strong co-localization with CDC42 in both cell lines (Figure 4D). It has been previously confirmed that RhoGDIa is a key negative regulator of $\mathrm{CDC} 42^{\mathrm{GTP}}[31,32]$. Our data suggest that, by stabilizing the complex of RhoGDIa and CDC42, 
NDRG1 plays a crucial role in keeping CDC42 in the inactive form and thereby prevents filopodia formation and cancer cell invasion. Once NDRG1 was lost, the cancer cells will develop into a more aggressive form with an excessively activated cytoskeleton-reorganization signaling axis.

A
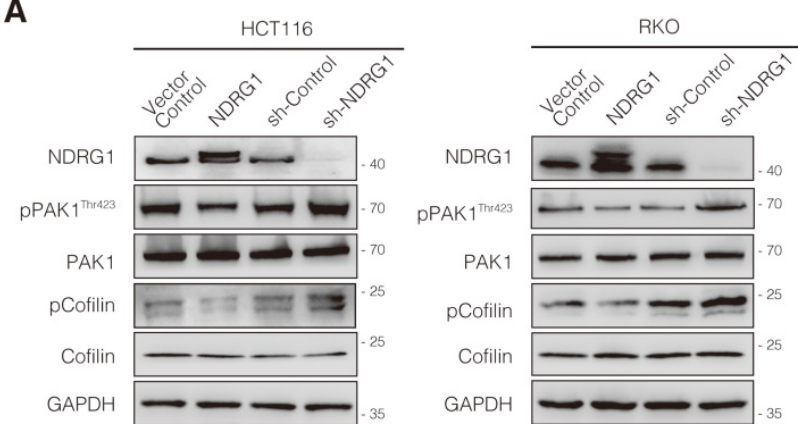

B

BCT116 GAPDH
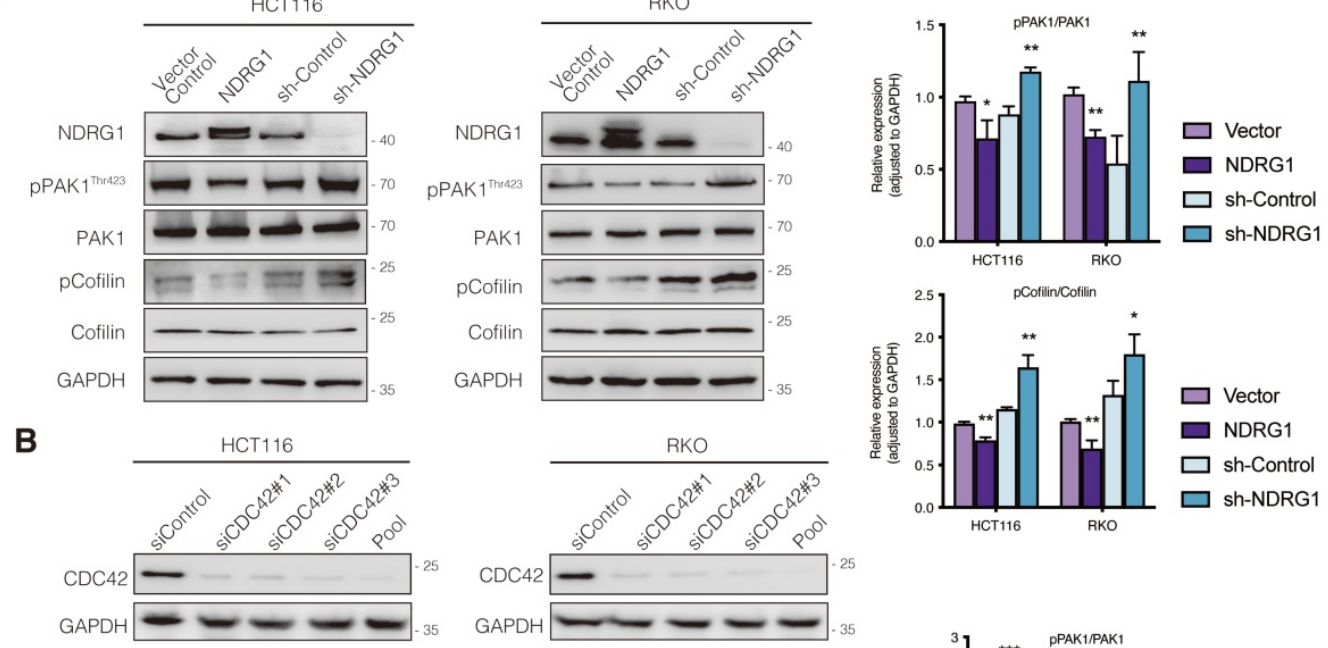

C
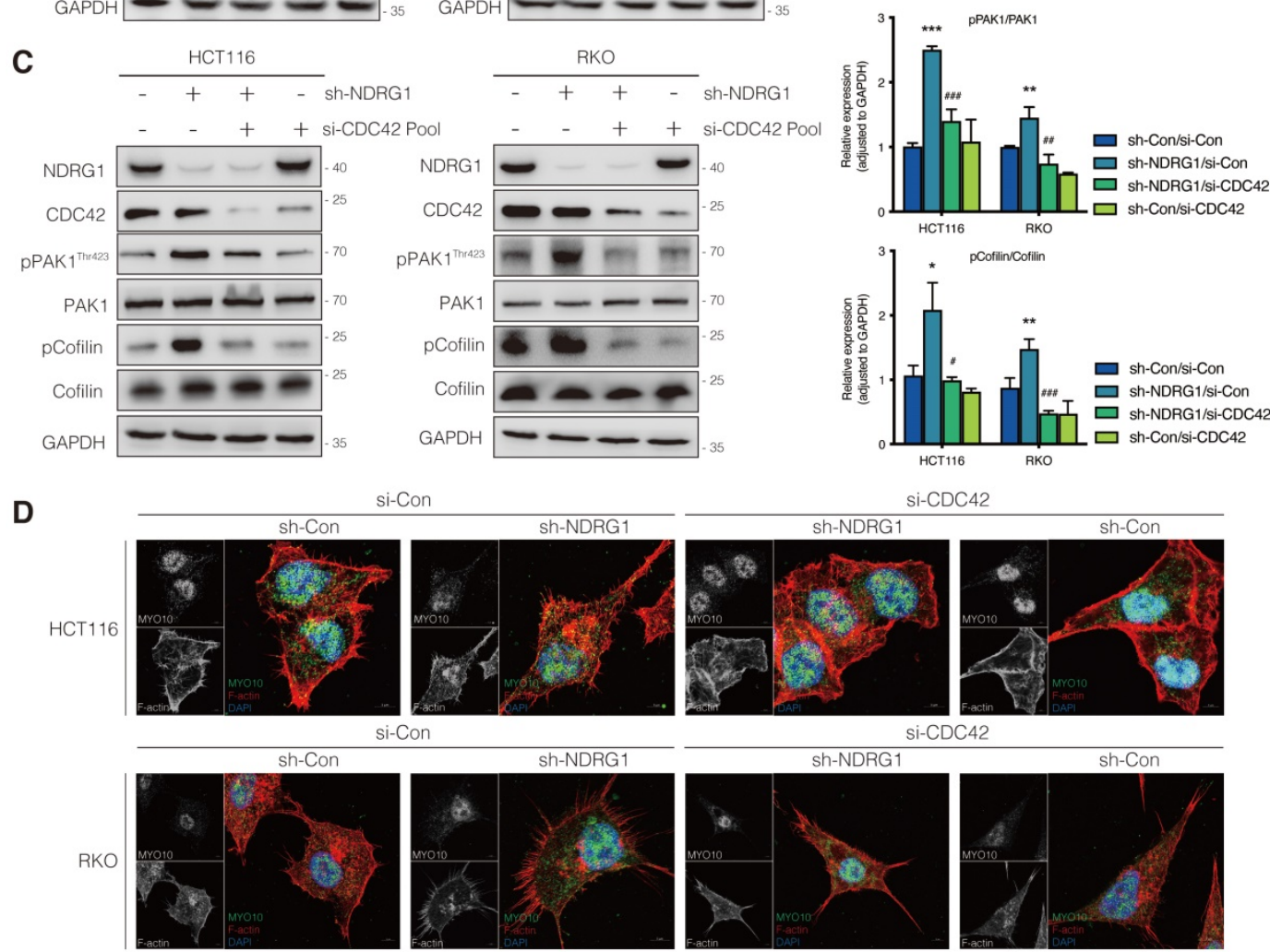

$\mathrm{i}-\mathrm{CDC} 42$
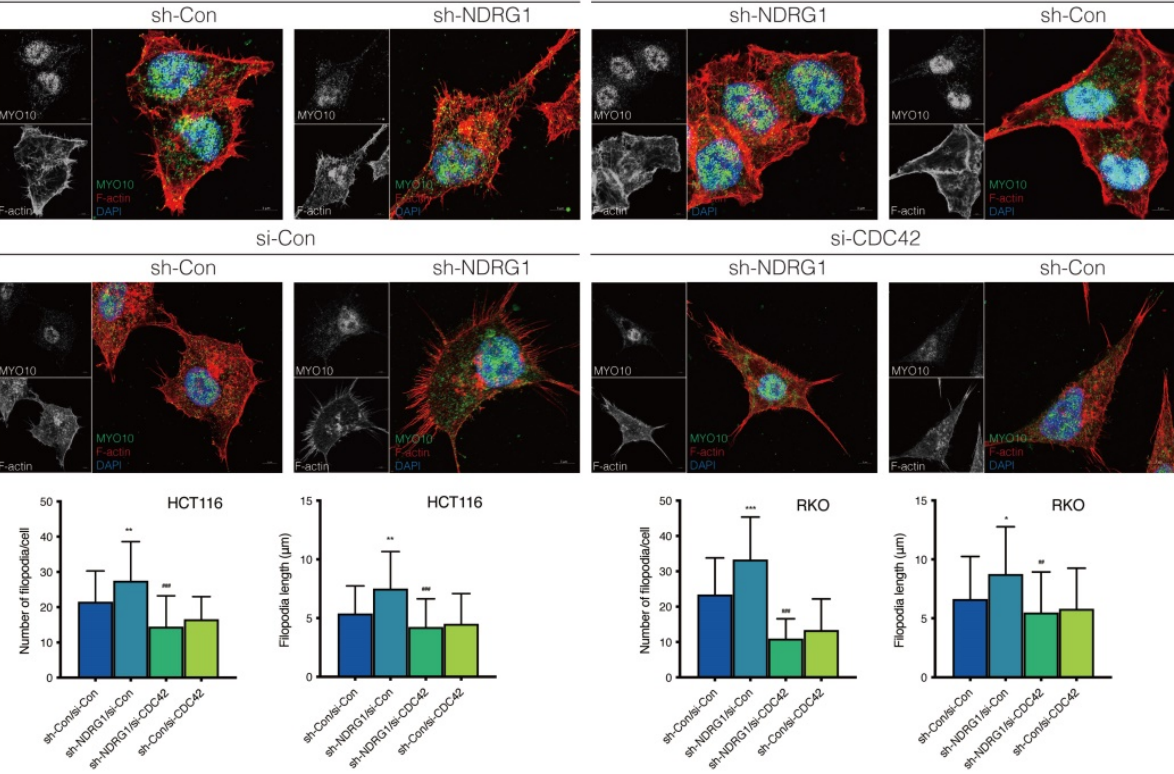

Figure 3. Inhibition of CDC42 prevents NDRG1 loss induced CRC cell filopodial protrusion formation through suppression of PAK1/Cofilin signaling. A) Immunoblotting analysis of the expression level of the total and phosphorylation form of PAK1 and Cofilin in indicated cell lines. B) Knockdown of CDC42 in HCT116 (left) and RKO (right) cells confirmed with immunoblotting analysis. Pool, combined siCDC42 sequences. C) Expression level of the total and phosphorylation form of PAK1 and Cofilin in indicated cell lines. D) Confocal images were taken to show immunofluorescence staining of MYO10 (green) and rhodamine-phalloidin (red) accompanied by the cell nucleus (blue) in colorectal cancer cells. Quantification of the MYO10-associated filopodial protrusions density and length is represented as mean \pm S.D.; results are representative of 3-5 images from different visual fields, $\mathrm{n}>50$ cells. $* \mathrm{P}$ value $<0.05$, **P value $<0.01$, ***P $<0.001$, relative to the sh-Con/si-Con groups. \#P value $<0.05$, \#P value $<0.01$, \#\#\# $<0.001$, relative to the sh-NDRG1/si-Con groups. 
A

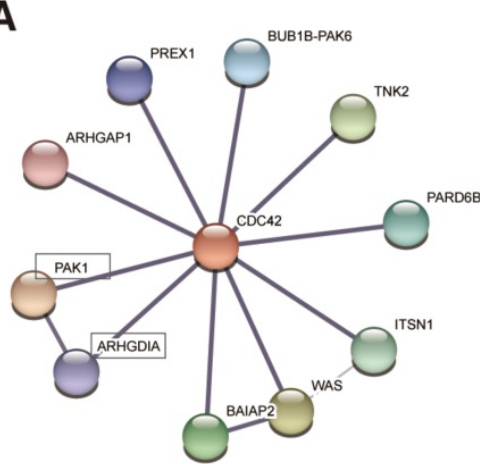

B
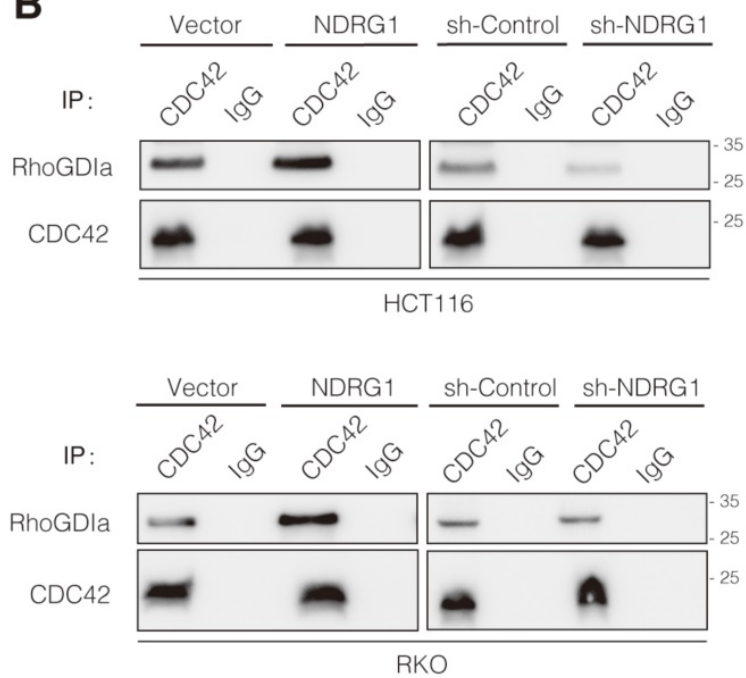

D

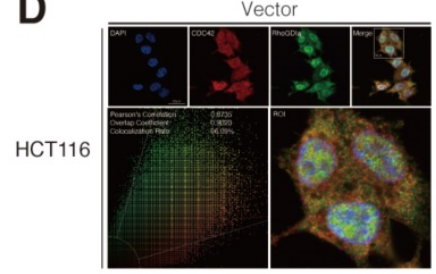

Vector

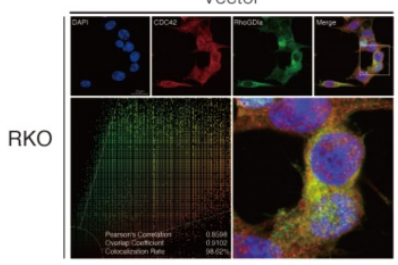

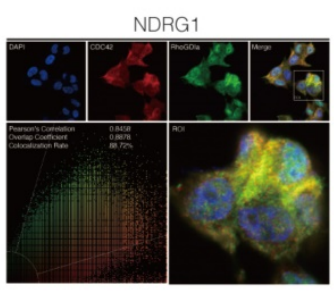

NDRG1

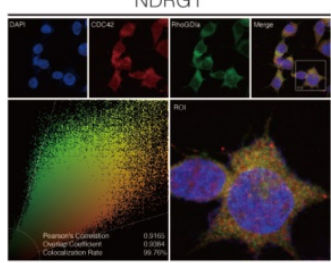

C

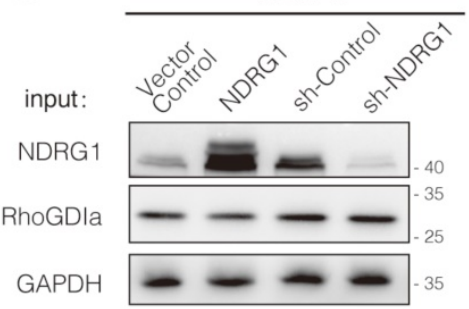

RKO
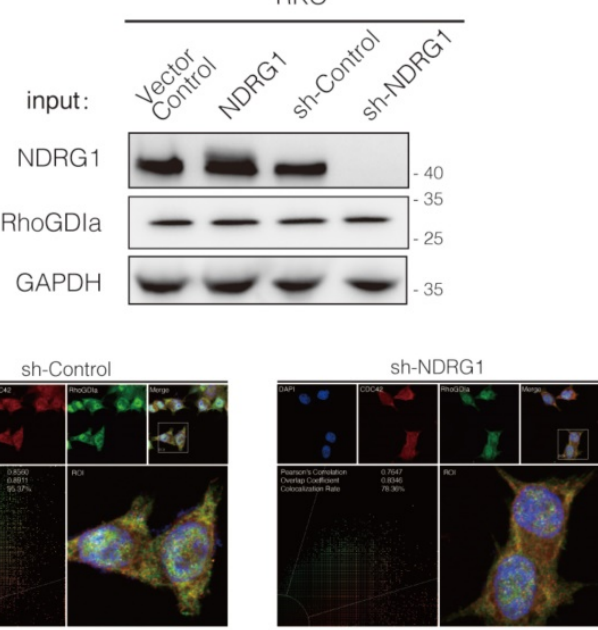

sh-NDRG1
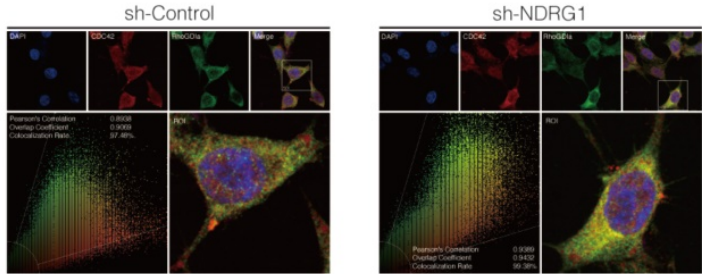

Figure 4. NDRG1 suppresses CDC42 activity by stabilizing the RhoGDla-CDC42 binding. A) The STRING network view of interactive proteins of CDC42 in humans. Gray lines between the nodes indicate various types of interaction evidence. B) Co-immunoprecipitation to examine the interaction of RhoGDla and CDC42 in both HCT116 and RKO cell lines. C) Immunoblotting assay to evaluate the influence of NDRG1 modification on RhoGDla expression in indicated cells. GAPDH was used as loading control. D) Double stained confocal immunofluorescence assay and co-localization analysis to confirm the interaction of RhoGDla and CDC42 in indicated cells (red: CDC42, green: RhoGDla, blue: DAPI, scale bar: $20 \mu \mathrm{m}$ ). Co-localization analysis on wide-field merged images was performed via Leica Application Suite X. Results are representative of five images from different visual fields.

\section{NDRG 1-knockdown facilitates peritoneal dissemination of CRC cells and correlates with active CDC42 expression in vivo}

Given that the filopodium-like protrusions enable the outgrowth of cancer macrometastases at distal sites [33], we asked whether the depletion of NDRG1 could promote the peritoneal tumor macrometastases of colorectal cancer in vivo. We first engineered luciferase-green fluorescent protein (GFP)-tagged sh-NDRG1 and sh-Control HCT116 cells and injected them intraperitoneally into nude mice. Not surprisingly, the bioluminescent imaging showed the tumor burdens of the sh-NDRG1 group were more massive than that of controls in the peritoneum of hosts (Figure 5A-B, $\mathrm{P}<0.01$ ). 
Immunofluorescence staining of NDRG1 and CDC42 ${ }^{\mathrm{GTP}}$ was performed to examine further their correlation in disseminated peritoneal foci, which was first confirmed as CRC metastases by pathology (Figure 5C). The results demonstrated a higher expression of CDC42GTP in the NDRG1-knockdown group than the control (Figure 5D, $\mathrm{P}<0.05$ ). The above findings indicated that silencing NDRG1 facilitates peritoneal dissemination of colorectal cancer, and it is correlated with an elevated level of active CDC42.

\section{Active CDC42 was frequently upregulated in CRC and was negatively related to NDRG 1 expression}

In our previous study, NDRG1 was remarkably reduced in CRC tissues and was negatively correlated with tumor stage and metastasis [8]. According to our extensive literature review, studies involving active CDC42 expression in human colon cancers were rarely reported. To gain further insight into the manner of NDRG1 and CDC42GTP expression with clinicopathological parameters, we used tissue array containing 86 pairs of cancer and matched peritumor specimens of colorectal cancer patients. Consecutive slices from the same sample were applied in staining the two proteins (Figure 6A). We delimited the overall cohort of 86 patients into two groups by the median staining intensity of CDC42GTP and discovered more robust $\mathrm{CDC} 42^{\mathrm{GTP}}$ expression in more advanced cancer (e.i., T-stage was higher, Figure 6B, $\mathrm{P}=0.016$ ). Moreover, high CDC42GTP expression was associated with low NDRG1 expression, and vice versa $(\mathrm{P}=0.009)$. In the meantime, we assessed baseline information and other clinicopathological features, where the various intensity of CDC42GTP made no significant difference (Figure 6B). These results collectively indicated that CDC42GTP expression has a negative correlation with NDRG1 expression in CRC tissues and is associated with the advanced invasiveness of tumor.
A
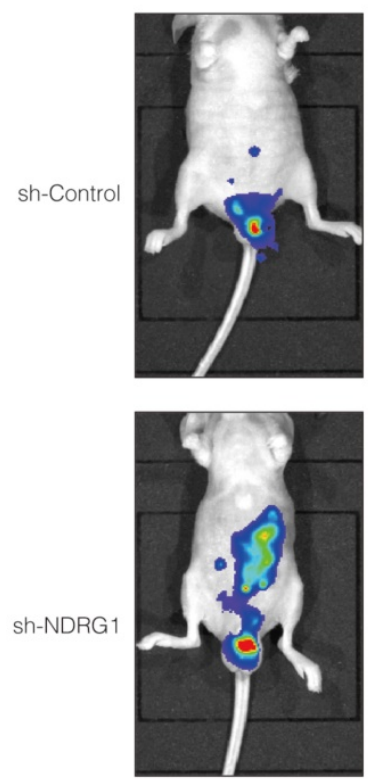

D
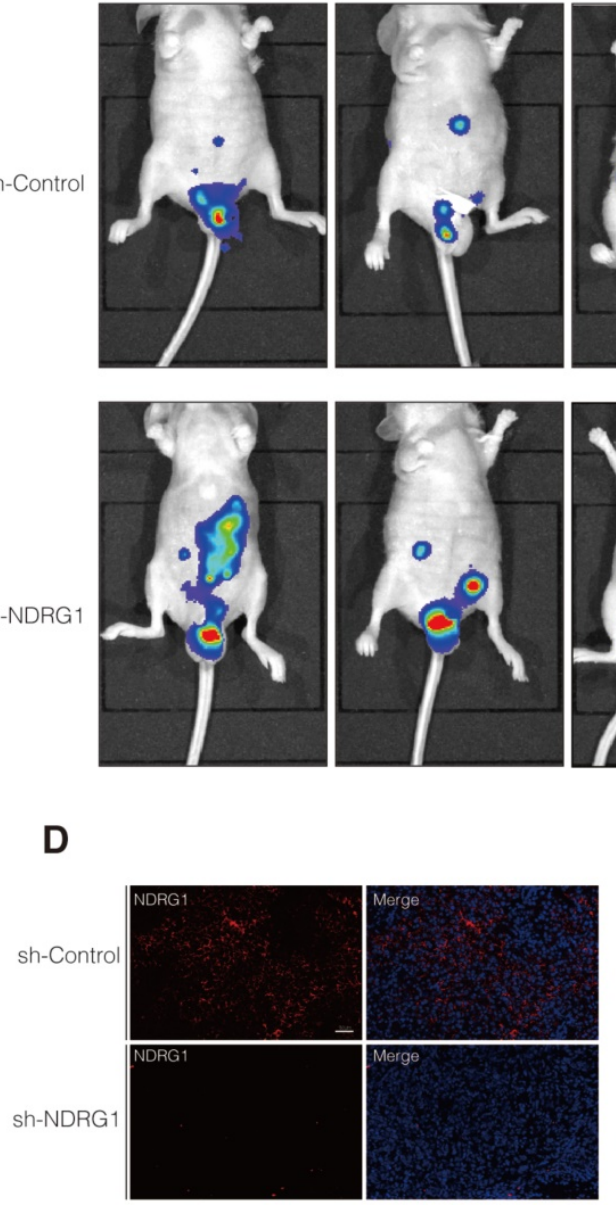
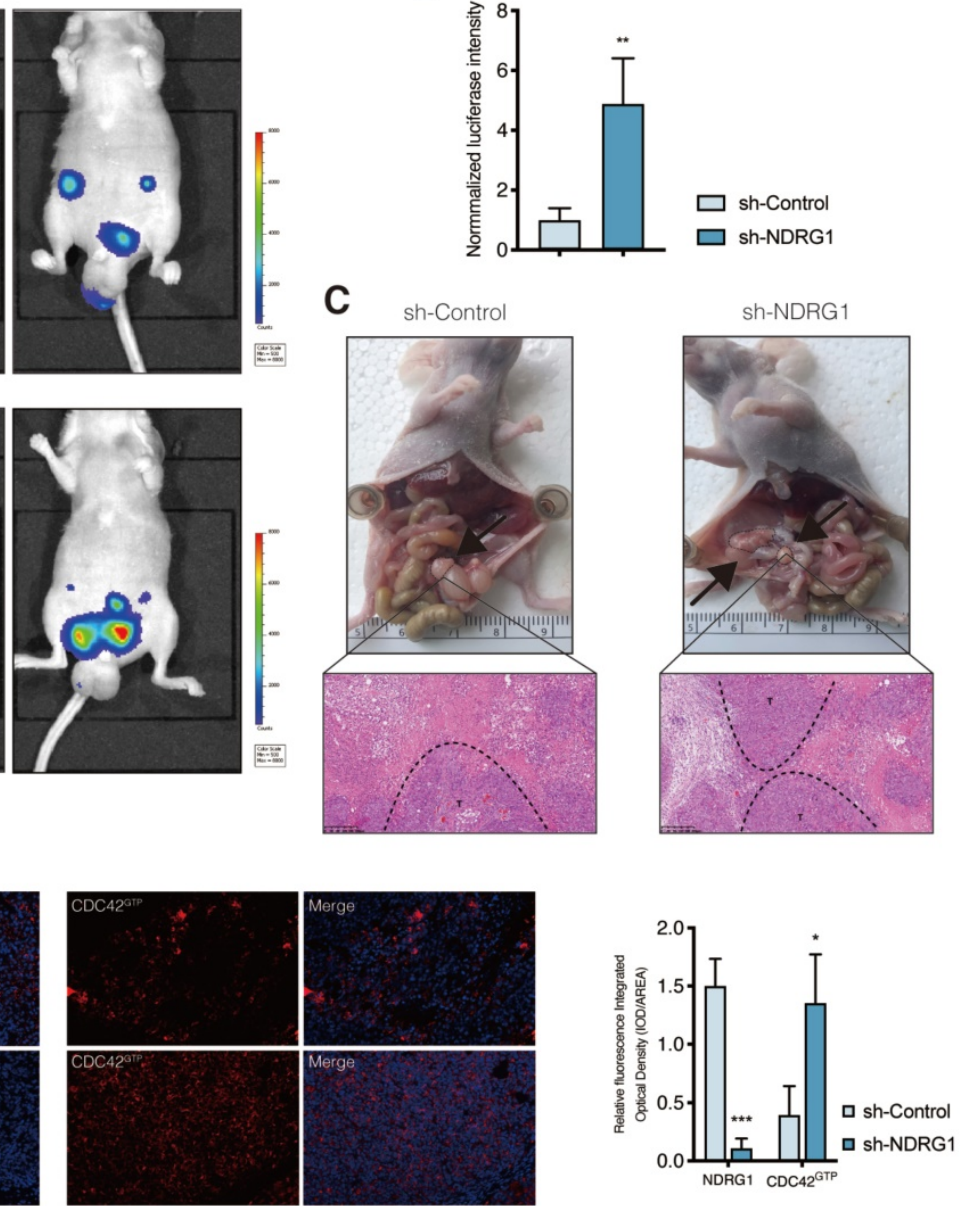

Figure 5. Silence of NDRG1 promotes the peritoneal metastasis and correlates with upregulated CDC42GTP expression. A) Peritoneal metastasis of CRC cells in BALB/c nude mice. Tumors in two groups were measured in situ and assessed by bioluminescence imaging in the fourth week. B) Statistical analysis of the bioluminescence in peritoneal foci of both groups. Results are shown as mean \pm S.D. C) Tumors in two groups are demonstrated after laparotomy with hematoxylin-eosin staining of peritoneal foci on the lower panel. Scale bars are as indicated. D) Immunofluorescence staining of NDRG1 (left) or CDC42GTP (right) accompanied by the cell nucleus stained by DAPI in peritoneal foci derived from sh-NDRG1 and control groups. Results are representative of 3-5 images from different visual fields and the histogram values are mean \pm S.D.; *P value $<0.05, * * * \mathrm{P}<0.001$, relative to the respective control groups. Scale bar: $50 \mu \mathrm{m}$. 
A
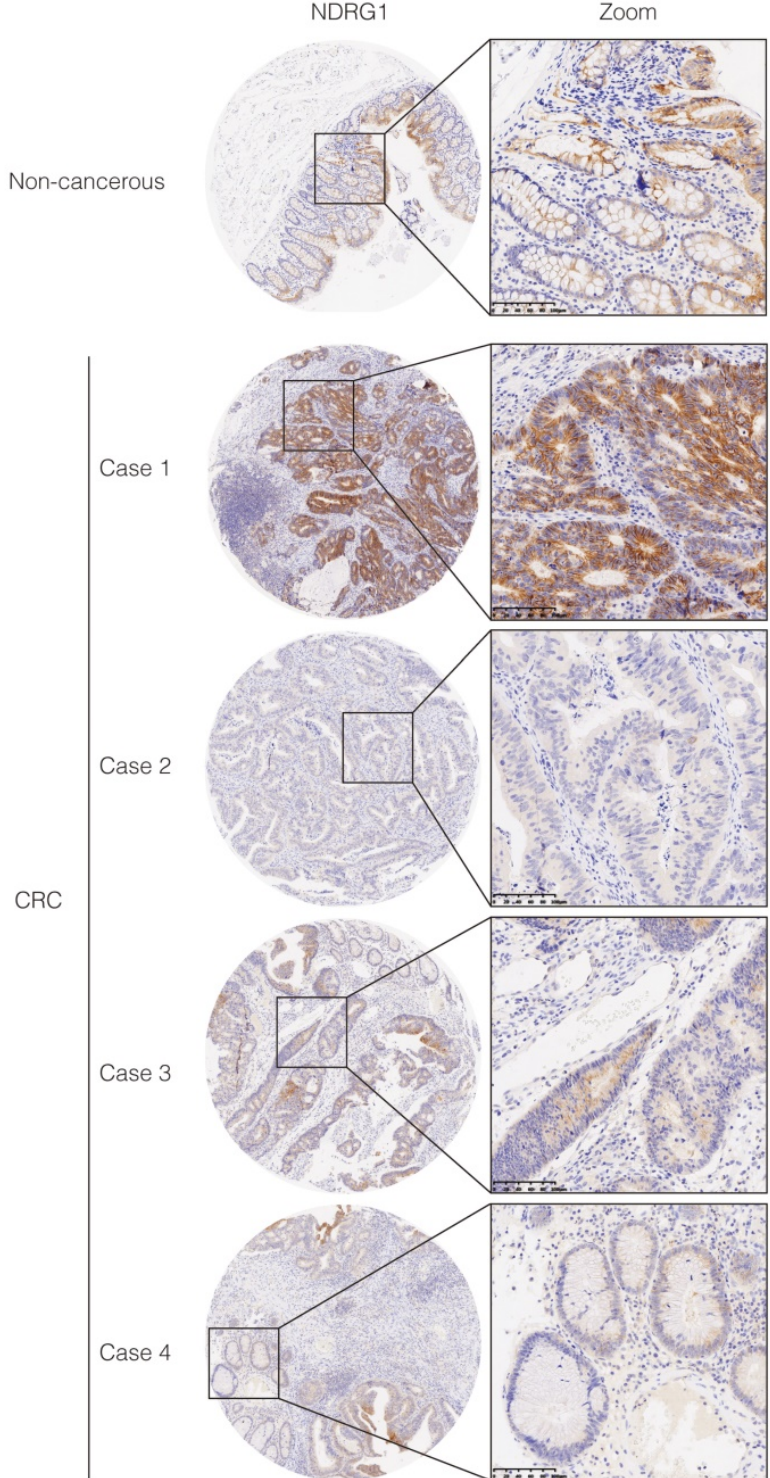

B

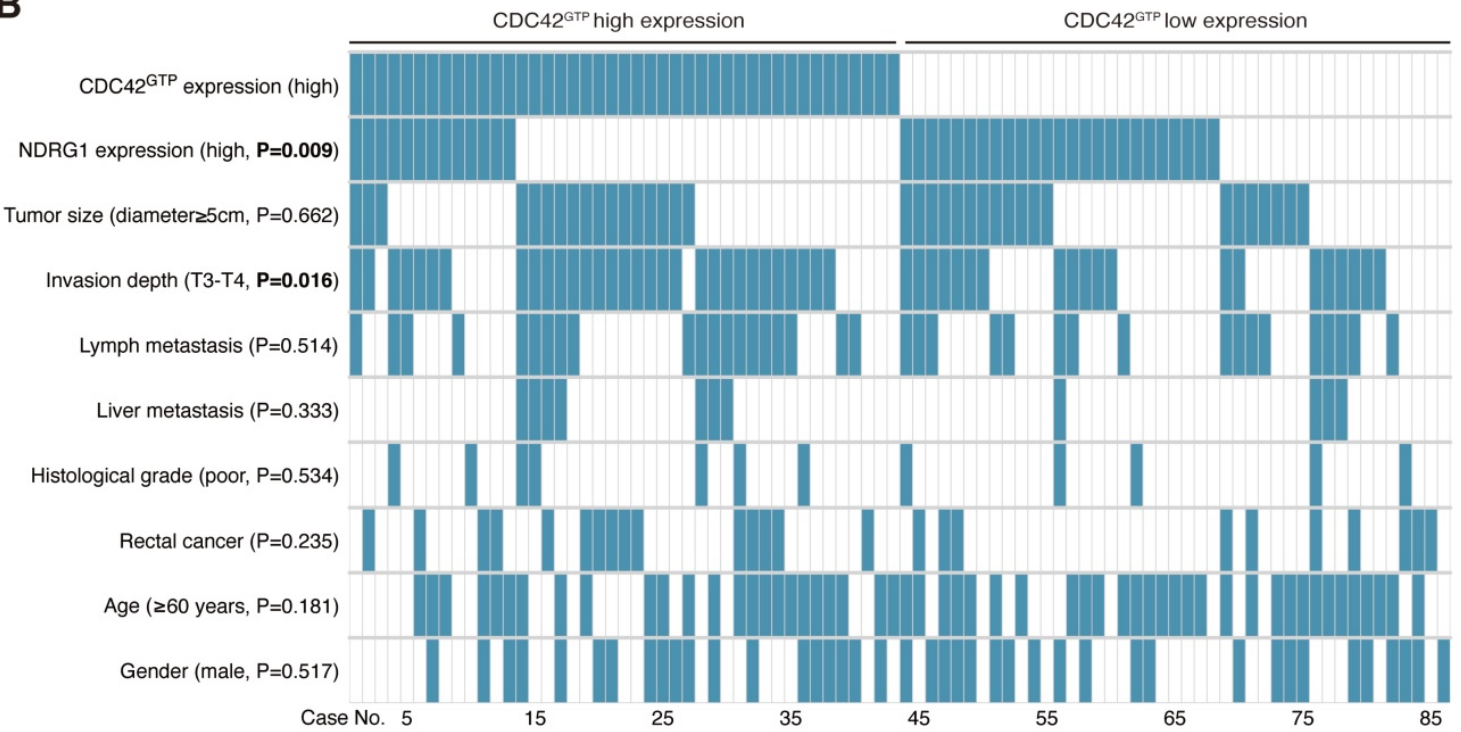

Figure 6. CDC42 ${ }^{\mathrm{TTP}}$ is frequently upregulated in CRC tissues and correlated with NDRG 1 expression and clinicopathological parameters. A) IHC staining of NDRG1 and active CDC42 expression in tumor and adjacent tissues in microarray. Magnification on the right with a scale bar of $100 \mu \mathrm{m}$. B) Heatmap illustrating different clinicopathological parameters between CDC42GTP-high and -low-expression tumors of the 86 cases. Statistical significance was analyzed by the $X^{2}$ test. $P$ values are as indicated. 


\section{Discussion}

In most malignancies, filopodia are associated with enhanced cancer cell invasion and metastasis and are a vital sign of actin-cytoskeleton reorganization. Based on our prior study that revealed the correlation of NDRG1 and stress fibers assembly [9] and the discovery of the potent mechanism of NDRG1 from public datasets (Figure S1), here we reported a novel mechanism of NDRG1 in regulating tumor invasion by mediating actin depolymerization in colorectal cancer. Using in vitro, in vivo, and tissue studies, we found that NDRG1 regulated actin cytoskeletal dynamics via modulating the activation of CDC42 and its downstream signaling pathway PAK1/Cofilin, which were realized by altering the stabilization of the RhoGDIa-CDC42 binding (Figure $7)$.

Filopodia, the actin-rich protruding projections that contribute to cell-cell communication [34], local invasion [35], directional migration [36], and cellular adhesions [37], are prominent features of invasive or immigrant cancer cells [38]. Similar to other cellular protrusions like podosomes which were reported to promote cancer invasiveness by degrading extracellular matrix [39], filopodia plays a profound role in tumor cell movement, orchestrates the

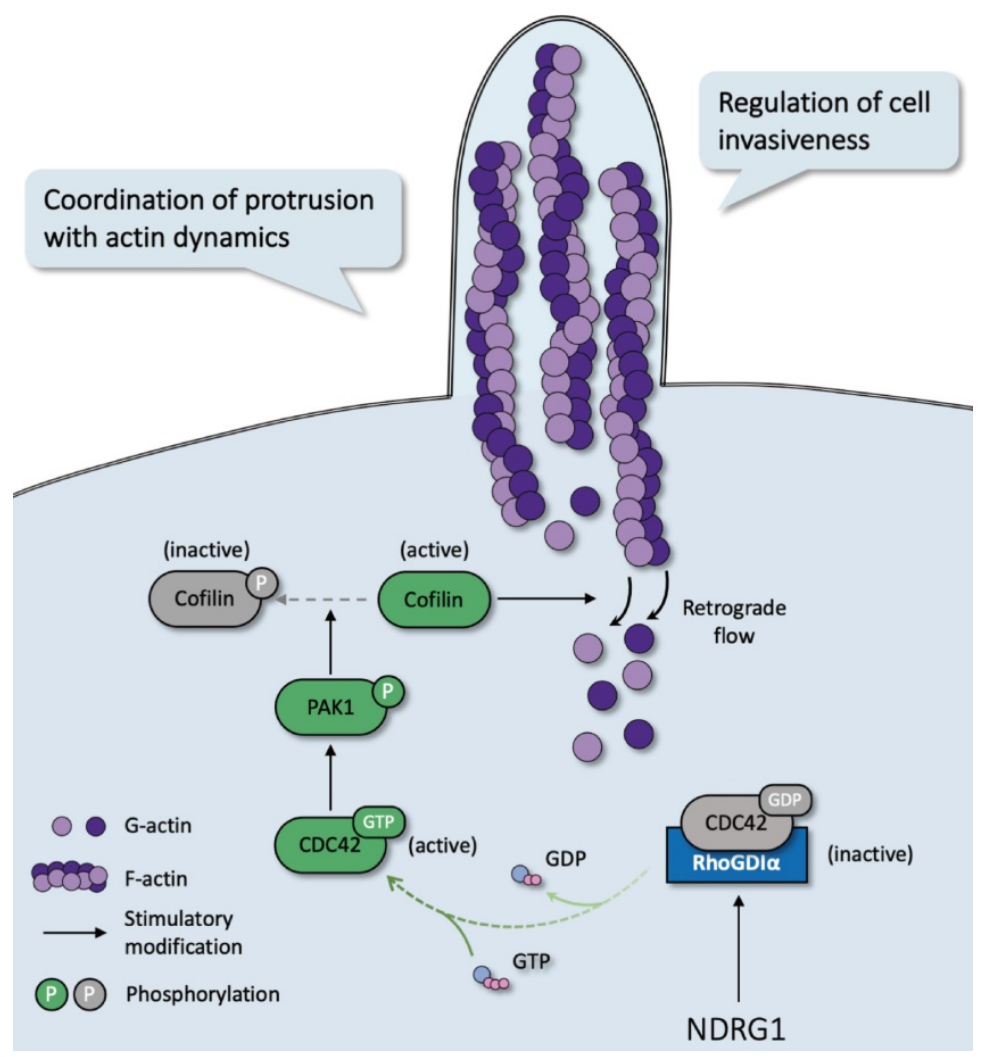

Figure 7. Schematic diagram for the mechanism of NDRG1's regulation of CDC42/PAK1/Cofilin axis as a switch that modulates actin cytoskeleton rearrangement in human colorectal cancer invasion by stabilizing the RhoGDIa-CDC42 binding. mesenchymal cell motility with its close coordination of adhesion at the leading edge [40]. This cell motility process occurs through two possible mechanisms: (1) actin polymerization against the cellular membrane at the leading edge provides force [3] and/or (2) actin filaments form stress fibers composed of contractile actomyosin structures [41, 42]. Cofilin promotes debranching and depolymerization of actin and promotes severing of the filaments into short segments [28], while phosphorylated Cofilin, an inactive form of the same protein, stops the disassembly of actin filaments [43]. Moreover, the PAKs family composed of 6 members is initially determined as protein kinases that function downstream of the Rho GTPases CDC42 and RAC [44]. PAK1, which belongs to Group I PAKs, is most often associated with carcinogenesis, where the PAK1/LIMK1/Cofilin signaling is frequently involved [29, 45, 46]. On account of their well-characterized roles in cancer, we investigated the mechanism of actin filament reorganization in colorectal cancer cells in an NDRG1-modified manner. We discovered that NDRG1 inhibits cell invasion via the effects on PAK1/Cofilin-regulated actin filament debranching (Figure 3). To the best of our knowledge, this work is the first to point out that NDRG1-modulated cytoskeleton rearrangement and invasion are obtained through regulating the CDC42 activation.

Persistent activation of Rho GTPase and the subsequently regulated genes that drive actin filament reorganization have been demonstrated to be pivotal for tumor progression and, therefore, negatively affect the prognosis of patients [47, 48]. Thus, targeting Rho GTPase and inhibiting the bypass signaling depicts a promising strategy for cancer therapy $[49,50]$. Our previous study revealed that NDRG1 exerts the inhibitory role in cell migration via Rho kinasemediated regulation of pMLC2 (phosphorylated myosin II light chain) [9]. Although Rho and CDC42 control actin-myosin contractility and the filopodia formation, respectively, there is convergence between the two Rho GTPases' signaling. For example, MRCK (myotonic dystrophy kinaserelated CDC42 binding kinase) and ROCK cooperate in the myosin-dependent cell motility [51], while CDC42 and RhoA could act antagonistically in regulating membrane metalloproteinase localization [52]. Moreover, Rho-ROCK activity is required in the motility and bleb-like protrusion formation in rounded tumor cells, like A375m2 melanoma, but not 
elongated F-actin-rich protrusive movement of cells like BE colon carcinoma [53]. El Sibai et al. also reported that the inhibition of ROCK leads to a switch between CDC42- and RAC1-dependant membrane protrusion in rat mammary adenocarcinoma cells [54]. In the present study, the observation of switched CDC42-activation using GST-pull down assay, a widely approved approach to accurately assess the activation of the Rho GTPase, has demonstrated a close relationship between CDC42 and NDRG1 (Figure 2).

Next, we exhibited that the inhibition of actin depolymerization via phosphorylation of PAK1/ Cofilin could be responsible for the NDRG1-regulated actin reorganization, which was further confirmed by abolishing CDC42 (Figure 3). The silencing of CDC42 blocked the phosphorylation of PAK1/Cofilin and further diminished the excessive stimulation of the filopodia formation motivated by NDRG1 knockdown. Importantly, this novel axis revealed in our study demonstrates a mechanism by which NDRG1 exerts the anti-invasion effects through remodeling the actin cytoskeleton in colorectal cancer. Further investigations on clinical tissue and peritoneal dissemination model of nude mice also indicated that CDC42 ${ }^{\mathrm{GTP}}$ expression has a negative correlation with NDRG1 expression in CRC tissues and is associated with the advanced invasiveness of the tumor (Figure $5-6)$.

The function of CDC42 and other Rho GTPase in tumorigenesis is thought to be positively related to cell surface receptors and interaction with GEFs, GAPs, and RhoGDIs [30, 55]. RhoGDIa is the most common and best-explained member of the family; it is ubiquitously expressed and interacts with several Rho GTPase [30] and functions as a crucial molecule that switches the oscillation of Rho GTPase between inactive and active states. The contact of RhoGDIa with GDP prevents its detachment from the GTPase or, contrarily, inhibits the binding of GTP and maintains the inactive state of CDC42 [55]. Through the Co-IP assay, we examined and proved the interaction of RhoGDIa and CDC42 was largely influenced in NDRG1-modified cells (Figure 4). When the binding of RhoGDIa to CDC42 increases in NDRG1-overexpression cells, it impedes the inappropriate activation of CDC42 and thereby inhibits the downstream signaling axis that controls cell morphology and polarization. Changes in the levels of expression of RhoGDIs have been correlated with several types of cancer, and feedback loops are coordinating the expression of both the Rho GTPases and RhoGDIs [56, 57]; however, little is known about how the expression and binding of RhoGDIa are regulated [30].
In summary, our current findings represent fundamental advances in the novel mechanism underlying NDRG1's anti-cancer character. Rho GTPase signaling is versatile and indispensable to cell adhesion and motility [58]. It is thus an important limitation that we evaluated the role of the two major Rho family members, CDC42 and RAC, in filopodia regulation and cell invasiveness, the effect of Rho and other actin-related molecules remains to be determined. Given the complicated crosstalk of Rho family GTPases, further investigation into the role of these genes and their interactions with NDRG1 is needed, and feedback regulating CDC42 oscillations and spatial self-organization remains to be solved. Further studies have yet to be carried out across more cancer types and in larger cohorts as well as in therapeutic agents, such as a novel iron chelator we are currently researching, to develop a more promising strategy in cancer therapies.

\section{Supplementary Material}

Supplementary figures.

http://www.ijbs.com/v17p1716s1.pdf

\section{Acknowledgements}

We are thankful to the Shanghai Institute of Traumatology and Orthopedics (Ruijin Hospital) for technical support in using the In vivo Imaging System (Caliper Life Science, USA). We are also grateful to Dr. Ying Huang and her team (Xiaoning Liu, Qin Hang) in the Core Facility of Basic Medical Sciences (Shanghai Jiao Tong University School of Medicine) for their excellent support in advanced microscope facilities and software.

\section{Funding}

The work was supported by the Natural Science Foundation of China (NSFC) (Grant NO. 81871984, 81572818).

\section{Authorship Contributions}

Batuer Aikemu: Conceptualization, resources, data curation, revising, software, formal analysis, validation, investigation, visualization, methodology, writing-original draft, revising, and editing.

Yanfei Shao: Resources, software, formal analysis, revising, validation, methodology, and editing.

Guang Yang: Resources, data curation, software, formal analysis, validation, investigation, methodology, writing-original draft, and editing.

Junjun Ma: Resources, formal analysis, methodology, and editing.

Sen Zhang: Resources, software, formal analysis, and methodology. 
Xiao Yang: Resources, investigation, visualization, and methodology.

Hiju Hong: Resources and methodology.

Galiya Yesseyeva: Analysis and validation.

Ling Huang: Formal analysis and validation.

Hongtao Jia: Resources.

Chenxing Wang: Revising and investigation.

$\mathrm{Lu}$ Zang: Conceptualization, data curation, formal analysis, supervision, acquisition, validation, methodology, investigation, and editing.

Jing Sun: Conceptualization, revising, data curation, formal analysis, supervision, acquisition, validation, methodology, investigation, writingoriginal draft, writing-review, and editing.

Minhua Zheng: Conceptualization, supervision, funding visualization, writing-original draft, project administration, validation, methodology, investigation, writing-review, and editing.

\section{Competing Interests}

The authors have declared that no competing interest exists.

\section{References}

1. Siegel RL, Miller KD, Jemal A. Cancer statistics, 2019. CA Cancer J Clin. 2019; 69: 7-34.

2. Chen H, Li N, Ren J, Feng X, Lyu Z, Wei L, et al. Participation and yield of a population-based colorectal cancer screening programme in China. Gut. 2019; 68: $1450-7$.

3. Pollard TD, Cooper JA. Actin, a central player in cell shape and movement. Science. 2009; 326: 1208-12.

4. Bae DH, Jansson PJ, Huang ML, Kovacevic Z, Kalinowski D, Lee CS, et al. The role of NDRG1 in the pathology and potential treatment of human cancers. J Clin Pathol. 2013; 66: 911-7.

5. Chen ZQ, Zhang DH, Yue F, Zheng MH, Kovacevic Z, Richardson DR. The Iron Chelators Dp44mT and DFO Inhibit TGF-beta-induced Epithelial-Mesenchymal Transition via Up-Regulation of N-Myc Downstream-regulated Gene 1 (NDRG1). Journal of Biological Chemistry. 2012; 287: 17016-28.

6. Sahni S, Bae DH, Lane DJR, Kovacevic Z, Kalinowski DS, Jansson PJ, et al. The Metastasis Suppressor, N-myc Downstream-regulated Gene 1 (NDRG1), Inhibits Stress-induced Autophagy in Cancer Cells. Journal of Biological Chemistry. 2014; 289: 9692-709.

7. Zhang $\mathrm{S}$, Yu C, Yang $\mathrm{X}$, Hong $\mathrm{H}, \mathrm{Lu} \mathrm{J}, \mathrm{Hu} \mathrm{W}$, et al. N-myc downstreamregulated gene 1 inhibits the proliferation of colorectal cancer through emulative antagonizing NEDD4-mediated ubiquitylation of p21. J Exp Clin Cancer Res. 2019; 38: 490.

8. Mi L, Zhu F, Yang X, Lu J, Zheng Y, Zhao Q, et al. The metastatic suppressor NDRG1 inhibits EMT, migration and invasion through interaction and promotion of caveolin-1 ubiquitylation in human colorectal cancer cells. Oncogene. 2017; 36: 4323-35.

9. Sun J, Zhang D, Zheng Y, Zhao Q, Zheng M, Kovacevic Z, et al. Targeting the metastasis suppressor, NDRG1, using novel iron chelators: regulation of stress fiber-mediated tumor cell migration via modulation of the ROCK1/pMLC2 signaling pathway. Mol Pharmacol. 2013; 83: 454-69.

10. Sharma A, Mendonca J, Ying J, Kim HS, Verdone JE, Zarif JC, et al. The prostate metastasis suppressor gene NDRG1 differentially regulates cell motility and invasion. Mol Oncol. 2017; 11: 655-69.

11. Lee JC, Chung LC, Chen YJ, Feng TH, Juang HH. N-myc downstreamregulated gene 1 downregulates cell proliferation, invasiveness, and tumorigenesis in human oral squamous cell carcinoma. Cancer Lett. 2014; 355: 242-52.

12. AJ R, MA S, K B, RA F, MH G, G B, et al. Cell migration: integrating signals from front to back. Science (New York, NY). 2003; 302: 1704-9.

13. Machesky LM. Lamellipodia and filopodia in metastasis and invasion. FEBS Lett. 2008; 582: 2102-11.

14. Shibue T, Brooks MW, Weinberg RA. An integrin-linked machinery of cytoskeletal regulation that enables experimental tumor initiation and metastatic colonization. Cancer Cell. 2013; 24: 481-98.

15. Bravo-Cordero JJ, Hodgson L, Condeelis J. Directed cell invasion and migration during metastasis. Current Opinion in Cell Biology. 2012; 24: 277-83.
16. Sanz-Moreno V, Gadea G, Ahn J, Paterson H, Marra P, Pinner S, et al. Rac activation and inactivation control plasticity of tumor cell movement. Cell. 2008; 135: 510-23.

17. Jacquemet $G$, Hamidi $H$, Ivaska J. Filopodia in cell adhesion, 3D migration and cancer cell invasion. Curr Opin Cell Biol. 2015; 36: 23-31.

18. Yang X, Zhang S, He C, Xue P, Zhang L, He Z, et al. METTL14 suppresses proliferation and metastasis of colorectal cancer by down-regulating oncogenic long non-coding RNA XIST. Mol Cancer. 2020; 19: 46.

19. Vital DL, Loarca-PiÒa G, Dia V, Mejia ED. Peptides extracted from common bean (Phaseolus vulgaris L.) non-digestible fraction caused differential gene expression of HCT116 and RKO human colorectal cancer cells. Food Research International. 2014; 62: 193-204.

20. Murphy DA, Courtneidge SA. The 'ins' and 'outs' of podosomes and invadopodia: characteristics, formation and function. Nat Rev Mol Cell Biol. 2011; 12: 413-26.

21. Schoumacher M, Goldman RD, Louvard D, Vignjevic DM. Actin, microtubules, and vimentin intermediate filaments cooperate for elongation of invadopodia. J Cell Biol. 2010; 189: 541-56.

22. Nobes CD, Hall A. Rho, rac, and cdc42 GTPases regulate the assembly of multimolecular focal complexes associated with actin stress fibers, lamellipodia, and filopodia. Cell. 1995; 81: 53-62.

23. Etienne-Manneville S, Hall A. Rho GTPases in cell biology. Nature. 2002; 420: 629-35.

24. Campellone KG, Welch MD. A nucleator arms race: cellular control of actin assembly. Nat Rev Mol Cell Biol. 2010; 11: 237-51.

25. DC E, LC S, GM B, GN G. Activation of LIM-kinase by Pak1 couples Rac/ Cdc42 GTPase signalling to actin cytoskeletal dynamics. Nat Cell Biol. 1999; 1: 253-9.

26. King CC, Gardiner EM, Zenke FT, Bohl BP, Newton AC, Hemmings BA, et al. p21-activated kinase (PAK1) is phosphorylated and activated by 3-phosphoinositide-dependent kinase-1 (PDK1). J Biol Chem. 2000; 275: 41201-9.

27. Breitsprecher D, Koestler SA, Chizhov I, Nemethova M, Mueller J, Goode BL, et al. Cofilin cooperates with fascin to disassemble filopodial actin filaments. J Cell Sci. 2011; 124: 3305-18.

28. Pollard TD, Borisy GG. Cellular motility driven by assembly and disassembly of actin filaments. Cell. 2003; 112: 453-65.

29. Jang I, Jeon BT, Jeong EA, Kim EJ, Kang D, Lee JS, et al. Pak1/LIMK1/Cofilin Pathway Contributes to Tumor Migration and Invasion in Human Non-Small Cell Lung Carcinomas and Cell Lines. Korean J Physiol Pharmacol. 2012; 16: 159-65.

30. Garcia-Mata R, Boulter E, Burridge K. The' invisible hand': regulation of RHO GTPases by RHOGDIs. Nat Rev Mol Cell Biol. 2011; 12: 493-504.

31. GF Z, YW X, J L, HL N, WX M, J X, et al. Mir20a/106a-WTX axis regulates RhoGDIa/CDC42 signaling and colon cancer progression. Nature communications. 2019; 10: 112 .

32. Hoffman GR, Nassar N, Cerione RA. Structure of the Rho Family GTP-Binding Protein Cdc42 in Complex with the Multifunctional Regulator RhoGDI. Cell. 2000; 100: 345-56.

33. Shibue T, Brooks MW, Inan MF, Reinhardt F, Weinberg RA. The outgrowth of micrometastases is enabled by the formation of filopodium-like protrusions. Cancer Discov. 2012; 2: 706-21.

34. Sagar, Pröls F, Wiegreffe C, Scaal M. Communication between distant epithelial cells by filopodia-like protrusions during embryonic development. Development. 2015; 142: 665-71.

35. Jacquemet G, Hamidi H, Ivaska J. Filopodia in cell adhesion, 3D migration and cancer cell invasion. Current Opinion in Cell Biology. 2015; 36: 23-31.

36. Gupton SL, Gertler FB. Filopodia: the fingers that do the walking. Sci STKE. 2007; 2007: re5.

37. Biswas $\mathrm{KH}$, Zaidel-Bar R. Early events in the assembly of E-cadherin adhesions. Exp Cell Res. 2017; 358: 14-9.

38. Petrie RJ, Yamada KM. At the leading edge of three-dimensional cell migration. Journal of Cell Science. 2012; 125: 5917-26.

39. Chen YW, Lai CS, Chen YF, Chiu WT, Chen HC, Shen MR. STIM1-dependent $\mathrm{Ca}(2+)$ signaling regulates podosome formation to facilitate cancer cell invasion. Sci Rep. 2017; 7: 11523.

40. O'Neill GM. The coordination between actin filaments and adhesion in mesenchymal migration. Cell Adh Migr. 2009; 3: 355-7.

41. Ong MS, Deng S, Halim CE, Cai W, Tan TZ, Huang RY, et al. Cytoskeletal Proteins in Cancer and Intracellular Stress: A Therapeutic Perspective. Cancers (Basel). 2020; 12.

42. Tojkander S, Gateva G, Lappalainen P. Actin stress fibers--assembly, dynamics and biological roles. J Cell Sci. 2012; 125: 1855-64.

43. Ichetovkin I, Han J, Pang KM, Knecht DA, Condeelis JS. Actin filaments are severed by both native and recombinant dictyostelium cofilin but to different extents. Cell Motil Cytoskeleton. 2000; 45: 293-306.

44. Rane CK, Minden A. P21 activated kinase signaling in cancer. Semin Cancer Biol. 2019; 54: 40-9.

45. Lv D, Li L, Lu O, Li Y, Xie F, Li H, et al. PAK1-cofilin phosphorylation mediates human lung adenocarcinoma cells migration induced by apelin-13. Clin Exp Pharmacol Physiol. 2016; 43: 569-79.

46. Pi J, Liu J, Zhuang T, Zhang L, Sun H, Chen X, et al. Elevated Expression of miR302-367 in Endothelial Cells Inhibits Developmental Angiogenesis via CDC42/CCND1 Mediated Signaling Pathways. Theranostics. 2018; 8: 1511-26. 
47. Ellenbroek SI, Collard JG. Rho GTPases: functions and association with cancer. Clin Exp Metastasis. 2007; 24: 657-72.

48. Zandvakili I, Lin Y, Morris JC, Zheng Y. Rho GTPases: Anti- or pro-neoplastic targets? Oncogene. 2017; 36: 3213-22.

49. Maldonado MDM, Dharmawardhane S. Targeting Rac and Cdc42 GTPases in Cancer. Cancer Res. 2018; 78: 3101-11.

50. Zhang Y, Li J, Lai XN, Jiao XQ, Xiong JP, Xiong LX. Focus on Cdc42 in Breast Cancer: New Insights, Target Therapy Development and Non-Coding RNAs. Cells. 2019; 8 .

51. Wilkinson S, Paterson HF, Marshall CJ. Cdc42-MRCK and Rho-ROCK signalling cooperate in myosin phosphorylation and cell invasion. Nat Cell Biol. 2005; 7: 255-61.

52. Ispanovic E, Serio D, Haas TL. Cdc42 and RhoA have opposing roles in regulating membrane type 1-matrix metalloproteinase localization and matrix metalloproteinase-2 activation. Am J Physiol Cell Physiol. 2008; 295: C600-10.

53. Sahai E, Marshall CJ. Differing modes of tumour cell invasion have distinct requirements for Rho/ROCK signalling and extracellular proteolysis. Nat Cell Biol. 2003; 5: 711-9.

54. El-Sibai M, Pertz O, Pang H, Yip SC, Lorenz M, Symons M, et al. RhoA/ROCK-mediated switching between Cdc42- and Rac1-dependent protrusion in MTLn3 carcinoma cells. Exp Cell Res. 2008; 314: 1540-52.

55. Cherfils J, Zeghouf M. Regulation of small GTPases by GEFs, GAPs, and GDIs. Physiol Rev. 2013; 93: 269-309.

56. Moissoglu K, McRoberts KS, Meier JA, Theodorescu D, Schwartz MA. Rho GDP dissociation inhibitor 2 suppresses metastasis via unconventional regulation of RhoGTPases. Cancer Res. 2009; 69: 2838-44.

57. Ding J, Huang S, Wu S, Zhao Y, Liang L, Yan M, et al. Gain of miR-151 on chromosome 8q24.3 facilitates tumour cell migration and spreading through downregulating RhoGDIA. Nat Cell Biol. 2010; 12: 390-9.

58. Cho HJ, Hwang YS, Yoon J, Lee M, Lee HG, Daar IO. EphrinB1 promotes cancer cell migration and invasion through the interaction with RhoGDI1. Oncogene. 2018; 37: 861-72. 